\title{
Efficient Contract Design in Multi-Principal Multi-Agent Supply Chains *
}

\author{
Thomas A. Weber ${ }^{\dagger} \quad$ Hongxia Xiong ${ }^{\ddagger}$
}

\begin{abstract}
We consider a general multi-principal multi-agent contracting game in a complete-information supply-chain setting and determine coordinating equilibrium transfer schedules in closed form. The resulting contracts manage to align incentives for decentralized decision-making and achieve first-best channel solutions. We allow for multidimensional actions and arbitrary payoff externalities between all members of the supply chain. For the coordinating contracts to exist it suffices that all payoff functions are continuous on the compact action sets in a general sense that accommodates discrete action sets. Our approach unifies and generalizes a significant portion of the extant supply-chain literature. It can be applied to a very large class of many-to-many supply-chain settings.
\end{abstract}

Keywords: Channel Coordination, Double Marginalization, Supply Chain Contracting.

${ }^{*}$ We would like to thank Hau Lee, William Lovejoy, Bruno Strulovici, Andrew Whinston, participants of the 2004 INFORMS Annual Meeting in Denver, CO, the 2006 INFORMS Annual Meeting in Pittsburgh, PA, the 2007 Annual Congress of the European Economic Association in Budapest, Hungary, the 2007 Annual Conference of the Production and Operations Management Society (POMS) in Dallas, TX, as well as seminar participants at Babson College, SFSU, Stanford University, University of Miami, University of Texas at Austin, and University of Washington for helpful comments. All remaining errors are our own.

${ }^{\dagger}$ Department of Management Science and Engineering, Stanford University. Terman Engineering Center, Stanford University, Stanford, CA 94305-4026. Phone: (650) 725-6827. Email: webert@stanford.edu. Research in part supported by a David Morgenthaler II Faculty Scholar Award.

${ }^{\ddagger}$ Department of Management Science and Engineering, Stanford University. Terman Engineering Center, Stanford University, Stanford, CA 94305-4026. Email: hxiong@stanford.edu. 


\section{Introduction}

In most modern supply chains a number of different organizations ("firms") contribute to the making and selling of products and services. In the eyes of the end consumers some of these products and services may be substitutes and others complements, which naturally leads to payoff interdependencies, at least for the competing firms downstream in the supply chain. Payoff interdependencies can also exist further upstream as a result of the firms' interactions on intermediate component markets. Due to legal restrictions of "anticompetitive behavior," horizontal interactions between different upstream or different downstream firms are typically confined to noncooperative market transactions, limiting the possibilities for explicit interfirm coordination. In contrast to this, many vertical relations in a supply chain are governed by nonmarket contractual mechanisms which by their very nature allow a high degree of interfirm coordination. Coordination in a supply chain is important, since it avoids efficiency losses due to double marginalization, which Spengler (1950) identified as a natural consequence of noncooperative behavior as long as the market price for end consumers reflects some monopoly power. Indeed, a supply chain is said to be "coordinated" if it maximizes the aggregate net payoffs of all firms involved. Our central research question is to identify contractual mechanisms that can be used to coordinate multi-principal multi-agent supply chains. The latter terminology suggests that supply chains - similar to firms (Jensen and Meckling 1976) - can be viewed as a nexus of contracts in which principals (as the designers of the contracts) propose appropriate individually rational and incentive-compatible mechanisms to their common agents. To capture some of the existing payoff externalities in supply chains, our model allows for a multitude of principals and agents, who engage in bilateral vertical contracting.

\section{$1.1 \quad$ Literature}

Fuelled by an increasing trend to outsource certain productive activities, contract design in supply chains has attracted great interest from practitioners and scholars alike. Cachon (2003) provides an excellent survey of the extant literature. The purpose of contract design generally consists in specifying a contractual mechanism that coordinates a given supply chain while all involved firms maintain control over their own actions. Most of the available results pertain to two-echelon singleprincipal single-agent supply chains in which the agent makes a single-dimensional decision. Coordinating solutions that have thus been proposed, including buy-back contracts (Pasternack 1985), revenue-sharing contracts (Cachon and Lariviere 2005), quantity-flexibility contracts (Tsay 1999), sales-rebate contracts (Taylor 2002), and quantity-discount contracts (Jeuland and Shugan 1983, Moorthy 1987), generally consist of parameterized reward schedules relating to the agent's action (e.g., order quantity, price, effort), as long as the latter is observable. ${ }^{1}$ Here, in addition to admitting multiple principals and multiple agents, which can be either upstream or downstream in

\footnotetext{
${ }^{1}$ In models with asymmetric information the agent's action may be hidden ("moral hazard") or either party may possess some private information ("hidden information"). We assume here that outcomes are contractible in the sense that they are both observable and verifiable by a third party. This presupposes a sufficiently high level of transparency in industries. It can be achieved (at least approximately) if monitoring costs are sufficiently low or if the contractual output can be sufficiently well specified and measured between parties.
} 
a two-echelon supply chain, we adopt a largely nonparametric approach with multidimensional actions.

In addition to the literature on serial two-echelon supply chains, there is some work in operations management dealing with multiple upstream firms and a single downstream firm (an "assembly system"), or, conversely, one upstream firm and multiple downstream firms (a "distribution system"). Carr and Karmarkar (2005) study competition in multi-echelon supply chains with an assembly system structure. They apply price-only contracts to achieve quantity coordination, i.e., the production quantity of each supplier (upstream firm) equals that of the manufacturer (downstream firm) who uses the suppliers' outputs as its own input in fixed proportions. However, their quantity coordination contract cannot achieve channel coordination. In a similar spirit, Majumder and Srinivasan (2003) consider competing supply chains each with a single supplier and multiple buyers ("supply trees"). The authors show that it is possible to coordinate the individual supply trees using two-part tariffs (i.e., a linear pricing schedule in addition to a fixed franchise fee). Bernstein and Federgruen (2005) investigate a distribution system with competing retailers and random demand and determine certain coordinating price-discount contracts. To the best of our knowledge, we are the first to consider the contract-design problem in a multi-principal multi-agent framework in an operations management setting. Indeed, as Cachon (2003) concludes, "[m]ore research is needed on how multiple suppliers compete for the affection of multiple retailers, i.e., additional emphasis is needed on many-to-one or many-to-many supply-chain structures."

In economics there has been work in several directions on principal-agent games. Bernheim and Whinston (1986) investigate a game of "common agency" (containing multiple principals and a single agent) under complete information. They show coalition-proof self-enforcing equilibria can be obtained by refining the set of Nash equilibria. Their "weakly truthful equilibria" are guaranteed to exist and yield efficient outcomes. Segal (1999) considers a game with one principal and multiple agents in which the agents' payoffs are interdependent. The author shows that as a result of the agents' payoff externalities an efficient Nash equilibrium with action-contingent transfers may not exist in this game. We show that Segal's result does not carry over to settings with with outcomecontingent transfers (cf. also footnote 12).

The more recent work by Prat and Rustichini (2003) (henceforth referred to as P\&R) studies a multi-principal multi-agent game (a "game played through agents") in which agents do not care about each others' actions. In that setting they are able to characterize pure-strategy equilibria of the contracting game and to provide a nonconstructive existence proof under the assumption that all payoff functions are concave. However, in practical supply-chain applications agent payoff externalities are ubiquitous and natural. For instance, manufacturers may contract with downstream retailers who compete in a common market and are thus affected by each others' actions. In addition, the retailers payoff functions may not necessarily be concave (e.g., when they face a convex demand curve). Completely dispensing with such restrictive assumptions, our approach generalizes the findings by $\mathrm{P} \& \mathrm{R}$ in several ways:

- First, we provide a characterization of weakly truthful equilibria allowing for externalities in all participants' payoffs. We show that any weakly truthful equilibrium outcome is efficient 
relative to its equilibrium transfer schedule, which does not imply the standard efficiency result that obtains in the absence of agent-payoff externalities.

- Second, we constructively provide a large set of closed-form contracts that coordinate a twotier supply chain in the presence of externalities by implementing any given efficient outcome as a weakly truthful equilibrium of the contracting game. The existence of these contracts depends solely on the continuity of the participants' payoff functions on the compact outcome sets. $^{2}$ The implementation is 'strong' (in the sense that agents strictly prefer efficient actions) whenever the efficient outcome is unique. Due to the agent-payoff externalities an equilibrium transfer schedule from a principal to an agent is outcome-contingent: it generally depends on all agents' actions, i.e., the full outcome of the game.

- Third, under the assumption that all participants' payoffs are concave we constructively provide a set of coordinating contracts with action-contingent transfer schedules: each transfer schedule proposed to an agent depends only on the action of that agent. We show that this set of contracts exists whenever the agents' aggregate 'compliance cost' is not too large. In particular, in the absence of agent-payoff externalities, this set of contracts can be affine for all principals except possibly one. We note that our results for that special case also generalize earlier results obtained by the authors and by Strulovici and Weber (2004).

The closed-form expressions of the coordinating equilibrium contracts contain state-contingent parameter functions that can be adjusted depending on the application at hand. In this paper we aim to bridge the gap between a practically useful operations management framework and the economics literature.

\subsection{Outline}

The rest of the paper is organized as follows. In Section 2 we first introduce the general model and the underlying equilibrium concept. We then focus our discussion on weakly truthful equilibria which yield efficient outcomes and thus coordinating contracts. Based on P\&R's work we characterize weakly truthful equilibria, discuss existence, and show that our framework applies to two-echelon supply chains with either supplier control or buyer control equally well. In Section 3 we study efficient contract design. For this, it is sufficient to consider a reduced contract-design problem any solution to which implies a solution to the original contract-design problem in terms of excess measures. We provide solutions to the reduced contract-design problem, each of which noncooperatively implements an efficient outcome using outcome-contingent transfer schedules. In the special case where all payoff functions are concave we provide a sufficient condition for the existence of a set of action-contingent transfer schedules that coordinate the supply chain. In Section 4 we discuss the application of our general method to the coordination of supply chains and compare the results to standard commercial contracts often used in practice. Finally, we conclude in Section 5 with directions for future research.

\footnotetext{
${ }^{2}$ If all agents' action sets are finite, then payoff continuity relative to these discrete sets is trivially satisfied and our results do apply.
} 


\section{The Model}

\subsection{Preliminaries}

Consider a setting in which principals can write outcome-contingent contracts with a number of different agents. By outcome we mean a vector of actions taken by the agents. ${ }^{3}$ Let $\mathcal{M}=\{1, \ldots, M\}$ and $\mathcal{N}=\{1, \ldots, N\}$ denote the corresponding sets of $M \geq 2$ principals and $N \geq 2$ agents. After each principal $m \in \mathcal{M}$ ("she") and each agent $n \in \mathcal{N}$ ("he") signs contracts with each other, ${ }^{4}$ agents noncooperatively implement an action (or "outcome") $x \in \mathcal{X}=\mathcal{X}_{1} \times \cdots \times \mathcal{X}_{N}$. The outcome vector $x=\left(x_{n}^{m}\right)_{m, n=1}^{M, N}$ contains each agent $n$ 's individual action vector $x_{n}=\left(x_{n}^{1}, \ldots, x_{n}^{M}\right) \in \mathcal{X}_{n} \subset \mathbb{R}_{+}^{M L}$ which in turn is composed of $M$ different $L$-dimensional actions $x_{n}^{m}$. His action set $\mathcal{X}_{n}$ is thereby a compact subset of $\mathbb{R}_{+}^{M L}$ which contains at least one point to allow for the possibility of inaction. In a supply-chain context it is useful to think of a "trade" $x_{n}^{m}$ as an $L$-dimensional vector of goods and services flowing from agent $n$ to principal $m$.

Each principal $m$ designs a mapping $t^{m}: \mathcal{X} \rightarrow \mathcal{T}^{m}$ from outcomes $x$ to transfer payments $t_{n}^{m}(x)$ directed at each agent $n \in \mathcal{N}$. The choice of principal $m$ 's transfer payment domain $\mathcal{T}^{m}$ thereby accommodates constraints reflecting the relationships between principals and agents, and we assume that $\mathcal{T}^{m}=\mathcal{T}_{1}^{m} \times \cdots \times \mathcal{T}_{N}^{m}$, where each $\mathcal{T}_{n}^{m}$ is either $\{0\}$ or $\mathbb{R}_{+}$. If no contractual relationship exists between principal $m$ and agent $n$ (cf. also Remark 2), then the $n$-th component of $t^{m}(x)$ could be constrained to vanish, i.e., $\mathcal{T}_{n}^{m}=\{0\}$, otherwise $\mathcal{T}_{n}^{m}=\mathbb{R}_{+}$.

\begin{tabular}{|l|l|}
\hline \hline Symbol & Explanation \\
\hline $\mathcal{M}=\{1, \ldots, M\}$ & Set of Principals \\
\hline $\mathcal{N}=\{1, \ldots, N\}$ & Set of Agents \\
\hline $\mathcal{X}=\mathcal{X}_{1} \times \cdots \times \mathcal{X}_{N}$ & Set of Feasible Outcomes/Actions \\
\hline $\mathcal{T}=\mathcal{T}^{1} \times \cdots \times \mathcal{T}^{M}, \mathcal{T}^{m}=\mathcal{T}_{1}^{m} \times \cdots \times \mathcal{T}_{N}^{m}$ & Set of Admissible Payments $\left(\mathcal{T}_{n}^{m} \in\left\{\{0\}, \mathbb{R}_{+}\right\}\right)$ \\
\hline$C(\mathcal{X}, \mathcal{T})$ & Set of Continuous Functions $f: \mathcal{X} \rightarrow \mathcal{T}$ \\
\hline$x=\left(x^{m}\right)_{m=1}^{M}=\left(x_{n}\right)_{n=1}^{N}=\left(x_{n}^{m}\right)_{m, n=1}^{M, N}$ & Feasible Outcome/Action $(x \in \mathcal{X})$ \\
\hline$t=\left(t^{m}\right)_{m=1}^{M}=\left(t_{n}\right)_{n=1}^{N}=\left(t_{n}^{m}\right)_{m, n=1}^{M, N}=\left(t_{n}^{m}, t_{-n}^{-m}\right)$ & Transfer Schedule $(t \in C(\mathcal{X}, \mathcal{T}))$ \\
\hline$\Delta=\left(\Delta_{n}^{m}\right)_{m, n=1}^{M, N}, \Delta^{m}=\sum_{n} \Delta_{n}^{m}, \Delta^{n}=\sum_{m} \Delta_{n}^{m}$ & Excess Transfer Schedule \\
\hline$V^{m} / v^{m} / F^{m}$ & Principal $m$ 's Net/Gross/Excess Payoff \\
\hline$U_{n} / u_{n} / G_{n}$ & Agent $n$ 's Net/Gross/Excess Payoff \\
\hline$W=\sum_{m} V^{m}+\sum_{n} U_{n}$ & Total Surplus \\
\hline \hline
\end{tabular}

Table 1: Some Notation.

Each agent $n$ cares about his action $x_{n} \in \mathcal{X}_{n}$ and the sum of all transfers he obtains in equilibrium. His net payoff is given by

$$
U_{n}(x ; t)=u_{n}(x)+\sum_{m \in \mathcal{M}} t_{n}^{m}(x)
$$

\footnotetext{
${ }^{3}$ We maintain the distinction between outcomes and actions throughout this paper.

4 "Selective contracting," where some principals do not sign contracts with some agents, is permitted.
} 


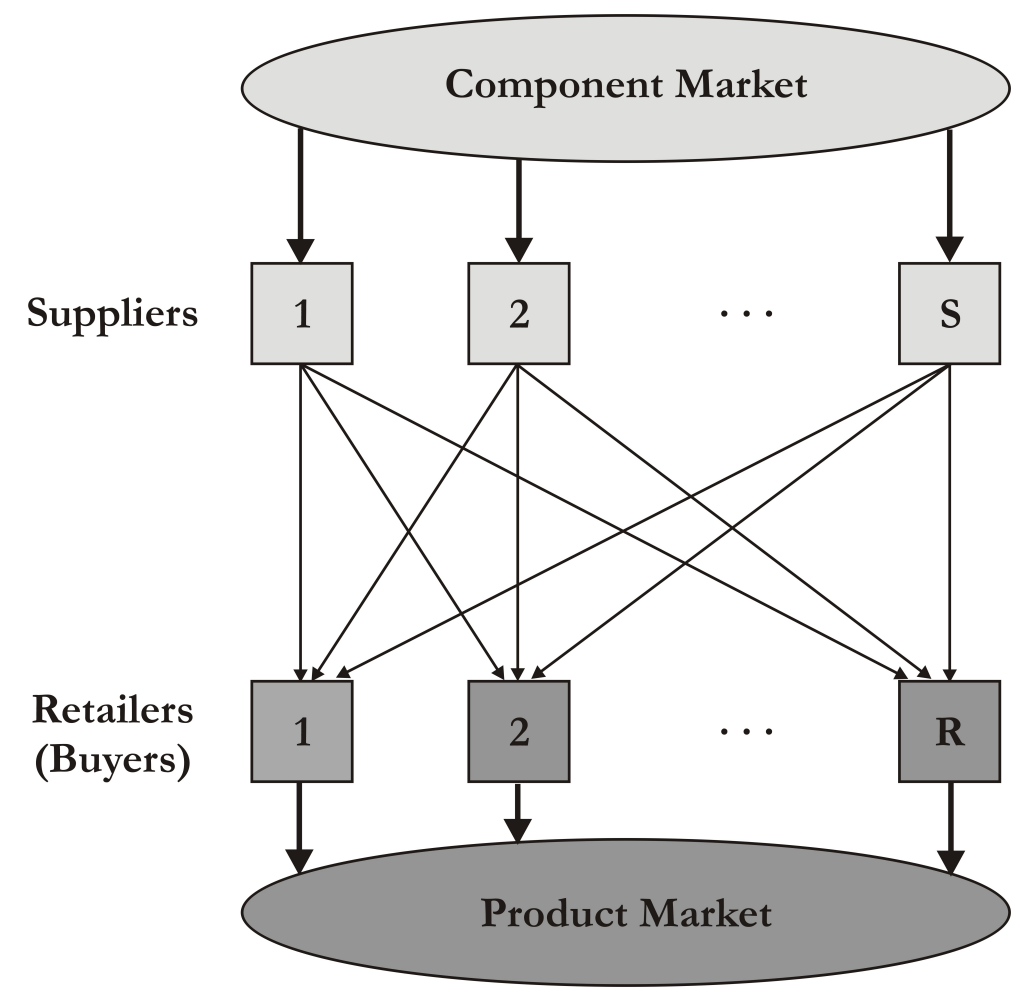

Figure 1: General Two-Echelon Supply-Chain Setup.

where $u_{n}(x)$ is the gross payoff to agent $n$ from the outcome $x=\left(x_{n}, x_{-n}\right)$ when he takes action $x_{n}$ and all other players implement $x_{-n}$.

When selecting optimal remuneration schemes (contracts) for the different agents, each principal cares about both her monetary payments and the agents' actions. Let $v^{m}(x)$ be principal $m$ 's gross payoff if action $x$ is taken. If she offers the transfer schedule $t^{m}=\left(t_{1}^{m}, \ldots, t_{N}^{m}\right)$ and agents implement the outcome $x$, her net payoff is

$$
V^{m}\left(x ; t^{m}\right)=v^{m}(x)-\sum_{n \in \mathcal{N}} t_{n}^{m}(x) .
$$

Our modelling framework is general enough to accommodate both positive and negative transfer payments corresponding to what we refer to as bottom-up (principals downstream) or top-down (principals upstream) contracting (cf. Section 2.5). In the terminology chosen by Grossman and Hart (1986) we will also refer to bottom-up contracting as "buyer control" and to top-down contracting as "supplier control." In this context we consider as our leading example a two-echelon supply chain consisting of $S$ suppliers and $R$ retailers (buyers) with retailers buying products from their upstream suppliers (cf. Figure 1). ${ }^{5}$ Depending on the balance of bargaining power in the supply chain, the retailers could act as either principals or agents. In bottom-up contracting, when retailer $m$ (as principal) buys the quantity $x_{n}^{m}$ of goods and services from supplier $n$ (as agent), we can expect $t_{n}^{m}$ to be positive and our framework exactly applies. In top-down contracting, when supplier $m$ (as principal) sells a quantity vector $x_{n}^{m}>0$ to retailer $n$ (as agent), we might expect

\footnotetext{
${ }^{5}$ If $\mathcal{S}=\{1, \ldots, S\}$ is the set of suppliers and $\mathcal{R}=\{1, \ldots, R\}$ is the set of retailers, then $(\mathcal{S}, \mathcal{R})=(\mathcal{M}, \mathcal{N})$ in the case of top-down contracting, whereas $(\mathcal{S}, \mathcal{R})=(\mathcal{N}, \mathcal{M})$ in the case of bottom-up contracting.
} 
the transfer $t_{n}^{m}$ from principal $m$ to agent $n$ to be negative, even though we earlier required that the payment domain $\mathcal{T}_{n}^{m}$ is a subset of $\mathbb{R}_{+}$. Nevertheless, we show in Section 2.5 that top-down contracting can be simply accommodated in the given framework by converting transfers into nonnegative payments ("quantity discounts" or "rebates") which are subtracted from a large enough transfer ("undiscounted wholesale price") in the opposite direction. Hence, the distinction between buying and selling, between bottom-up or top-down contracting, will prove insignificant for the results in this paper.

\subsection{Equilibrium Concept}

The sequence of events is as follows. First, each principal offers her vector of transfer-payment schedules to all agents simultaneously and noncooperatively. The transfer-payment schedules are publicly announced to all agents. ${ }^{6}$ Second, the agents noncooperatively implement their most preferred actions. A pure-strategy equilibrium of the two-stage contracting game

$$
\Gamma=\left\{\{\mathcal{M}, \mathcal{N}\},\left\{V^{m}(\cdot), U_{n}(\cdot)\right\},\left\{C\left(\mathcal{X}, \mathcal{T}^{m}\right), \mathcal{X}_{n}\right\}\right\}
$$

is a subgame-perfect Nash equilibrium in which all principals and agents use pure strategies.

Definition 1 A pure-strategy equilibrium of the game $\Gamma$ is a pair $(\hat{t}, \hat{x}) \in C(\mathcal{X}, \mathcal{T}) \times \mathcal{X}$ in which (i) for every $n \in \mathcal{N}$ given any $t \in C(\mathcal{X}, \mathcal{T})$ it is

$$
\hat{x}_{n}(t) \in \arg \max _{x_{n} \in \mathcal{X}_{n}} U_{n}\left(x_{n}, \hat{x}_{-n} ; t\right),
$$

and (ii) for every $m \in \mathcal{M}$ given $\hat{t}^{-m} \in C\left(\mathcal{X}, \mathcal{T}^{-m}\right)$ the relation

$$
\hat{t}^{m} \in \arg \max _{t^{m} \in C\left(\mathcal{X}, \mathcal{T}^{m}\right)} V^{m}\left(\hat{x}\left(t^{m}, \hat{t}^{-m}\right) ; t^{m}\right)
$$

holds.

We limit our analysis to pure-strategy equilibria of $\Gamma$. If the equilibrium contracts achieve coordination of the supply chain, they implement, by definition, an efficient outcome (Cachon 2003).

Definition 2 (i) The outcome $x^{*} \in \mathcal{X}$ is efficient ${ }^{7}$ if

$$
W\left(x^{*}\right) \geq W(x)
$$

for all $x \in \mathcal{X}$, where $W(x)=\sum_{m \in \mathcal{M}} v^{m}(x)+\sum_{n \in \mathcal{N}} u_{n}(x)$ corresponds to the system welfare contingent on outcome $x$. (ii) The outcome $x^{\circ} \in \mathcal{X}$ is efficient relative to the transfer schedule $t$ if

$$
W\left(x^{\circ}\right) \geq \sum_{m \in \mathcal{M}} V^{m}(x ; t)+\sum_{n \in \mathcal{N}} U_{n}\left(x_{n}, x_{-n}^{\circ} ; t\right)
$$

for all $x \in \mathcal{X}$.

\footnotetext{
${ }^{6}$ The case in which contracting is bilateral and each agent is only informed about his own contract terms with a particular principal is more delicate (cf. Segal and Whinston (2003) for an analysis of such a situation with one principal and $n$ agents).

${ }^{7}$ By "efficient" we mean that the aggregate benefits of principals and agents (constituting the producer system) are maximized excluding end consumers (on the product market) and further upstream suppliers (on the component market) whose benefits we consider as exogenous to the principal-agent system.
} 
The set of all efficient outcomes for a given supply chain (i.e., given the principals' and agents' payoff functions) thus corresponds to the set of maximizers of the total surplus $W$ on $\mathcal{X}$. The set of efficient outcomes relative to a transfer schedule contains all outcomes for which principals and agents cannot do better in aggregate provided that (i) each agent takes the action of other agents as given ("noncooperation"), and (ii) principals and agents must agree on the implemented outcome ("fulfilled expectations"). Without externalities in the agents' gross payoffs one can show that principals have no interest in having transfers to any agent depend on anything other than that agent's action, so that there are also no externalities in the agents net payoffs. ${ }^{8}$ As a result, in that situation the concepts of efficiency and relative efficiency coincide.

In what follows, we assume that there is a consensus about which particular efficient outcome $\hat{x}$ is to be implemented. In other words, parties should be able to communicate about (i.e., coordinate on) the outcome. In the special case when all parties' payoff functions are strictly concave (cf. Assumption 2 below) and the set of implementable outcomes $\mathcal{X}$ is convex, there exists a unique efficient outcome $\hat{x}$.

Since we are considering a game with multiple principals, it is necessary to take into account the possibility of coalition formation among principals, since such coalitions may form noncooperatively, i.e., without binding contracts between the principals. Such coalitional games have first been considered by Von Neumann and Morgenstern (1944), who also coin the notion of a "stable equilibrium," which is such that all players want to join a coalition only if the resulting payoffs are not dominated by any coalitional deviation. Bernheim et al. (1987) introduce (via a recursive definition) the notion of coalition-proof Nash equilibrium, which is self-enforcing among the members of any coalition. As Bernheim and Whinston (1986) demonstrate, to guarantee coalition proofness of a Nash equilibrium (in a game with multiple principals and one agent), it is sufficient to guarantee that there are no profitable coalitional deviations, which is achieved at what they term truthful equilibria. P\&R, while restricting attention to efficient outcomes, apply this notion to a game played through agents with multiple principals, analogous to the one considered here. It is the latter definition that we choose to adopt.

Definition 3 Principal $m$ 's transfer $t^{m} \in C\left(\mathcal{X}, \mathcal{T}^{m}\right)$ is weakly truthful relative to the outcome $\hat{x} \in$ $\mathcal{X}$ if $V^{m}\left(\hat{x} ; t^{m}\right) \geq V^{m}\left(x ; t^{m}\right)$ for all $x \in \mathcal{X}$.

If the principals' equilibrium transfers are all weakly truthful relative to the outcome $\hat{x}$ (not necessarily assumed to be efficient), then no principal would prefer to implement a different outcome with her transfer. Correspondingly, an equilibrium where all the principals' transfers are weakly truthful with respect to the same outcome must be self-enforcing.

Definition 4 A weakly truthful equilibrium (WTE) of the game $\Gamma$ is a pair $(\hat{t}, \hat{x})$ that is a purestrategy equilibrium of $\Gamma$ with outcome $\hat{x}$ and in which the transfer $\hat{t}^{m}$ of every principal $m \in \mathcal{M}$ is weakly truthful relative to $\hat{x}$.

\footnotetext{
${ }^{8}$ In an earlier version of this manuscript we have proved that this claim extends to the situation in which the agents' payoff externalities are additively separable, as then at the margin each agent is indifferent about any other agent's action.
} 
The notion of weak truthfulness is directly related to supply-chain coordination, since any outcome that is part of a weakly truthful equilibrium must be efficient relative to the equilibrium transfer. As pointed out earlier, the definitions of relative efficiency and efficiency coincide in the absence of agent payoff externalities, and in that special case Lemma 1 coincides with an earlier result by P\&R (Proposition 3).

Lemma 1 The outcome $\hat{x}$ of a weakly truthful equilibrium $(\hat{t}, \hat{x})$ is efficient relative to the equilibrium transfer schedule $\hat{t}^{9}$

Since we are interested in supply-chain coordination, we limit our attention to weakly truthful equilibria, for which - it turns out - there exists a simple and useful characterization.

\subsection{Characterization of Weakly Truthful Equilibria}

As Bernheim and Whinston (1986) and subsequently P\&R indicate, it is sufficient to consider nonnegative transfer payments when considering weakly truthful equilibria. Nonnegative transfer payments are natural in a bottom-up contracting situation with buyer control, as then typically positive payments are made for any goods flowing from suppliers to buyers. Nevertheless, by a simple change of variables it is possible to equivalently formulate the contracting problem with nonnegative transfers in a top-down contracting situation with supplier control, as is shown in Section 2.5. The following characterization of WTEs, which generalizes P\&R (in the sense that we allow for arbitrary agent payoff externalities), is later used to find contracts that implement a given efficient outcome $\hat{x}$.

Theorem 1 (Characterization of a WTE) A pair $(\hat{t}, \hat{x})$ arises in a weakly truthful equilibrium if and only if the following three conditions are satisfied:

$$
v^{m}(\hat{x})-\sum_{n \in \mathcal{N}} \hat{t}_{n}^{m}(\hat{x}) \geq v^{m}(x)-\sum_{n \in \mathcal{N}} \hat{t}_{n}^{m}(x)
$$

for every principal $m \in \mathcal{M}$ and every outcome $x \in \mathcal{X}$;

$$
u_{n}(\hat{x})+\sum_{m \in \mathcal{M}} \hat{t}_{n}^{m}(\hat{x}) \geq u_{n}\left(x_{n}, \hat{x}_{-n}\right)+\sum_{m \in \mathcal{M}} \hat{t}_{n}^{m}\left(x_{n}, \hat{x}_{-n}\right)
$$

for every agent $n \in \mathcal{N}$ with arbitrary action $x_{n} \in \mathcal{X}_{n}$; and

$$
u_{n}(\hat{x})+\sum_{i \in \mathcal{M}} \hat{t}_{n}^{i}(\hat{x})=\max _{x_{n} \in \mathcal{X}_{n}}\left\{u_{n}\left(x_{n}, \hat{x}_{-n}\right)+\sum_{i \neq m} \hat{t}_{n}^{i}\left(x_{n}, \hat{x}_{-n}\right)\right\},
$$

for every principal $m \in \mathcal{M}$ and every agent $n \in \mathcal{N}$.

\footnotetext{
${ }^{9}$ Actually, $(\hat{t}, \hat{x})$ a WTE implies that $W(\hat{x}) \geq\left(\sum_{m \in \mathcal{M}} \max _{\xi \in \mathcal{X}} V^{m}(\xi ; t)\right)+\left(\sum_{n \in \mathcal{N}} \max _{\xi_{n} \in \mathcal{X}_{n}} U_{n}\left(\xi_{n}, \hat{x}_{-n} ; t\right)\right)$, which implies relation (7). This stronger result is demonstrated in the proof of Lemma 1.
} 
The intuition of the equilibrium characterization in Theorem 1 is as follows. The weak truthfulness requirement in Definition 3 can be rewritten in the form (WT). Given the principals' equilibrium transfer schedules $\hat{t}^{1}, \ldots, \hat{t}^{M}$, the agents implement a Nash equilibrium. In other words, the outcome $\hat{x}=\left(\hat{x}_{1}, \ldots, \hat{x}_{N}\right)$ must be composed of elements of the agents' respective best-response correspondences, which is equivalent to requiring that (AM) ("agent payoff maximization") holds. Finally, in equilibrium each principal chooses her transfer schedules such as to minimize the cost of implementing the outcome $\hat{x}$. In other words, since transfers are nonnegative by assumption, principal $m$ has to pay agent $n$ not more than this agent would obtain by implementing his otherwise optimal action given that $t_{n}^{m}=0$ and all other principals' reward functions stay in place. This is principal $m$ 's "cost minimization" condition (PM) with respect to agent $n$.

\subsection{Existence of an Efficient Weakly Truthful Equilibrium}

We now discuss the existence of an efficient WTE with outcome-contingent and action-contingent transfer schedules. Note first that an efficient WTE is a WTE $(\hat{t}, \hat{x})$ that implements an efficient outcome $\hat{x} \in \arg \max _{x \in \mathcal{X}} W(x)$. The following assumption of continuous payoffs guarantees the existence of an efficient outcome. ${ }^{10}$

Assumption 1 (Payoff Continuity) The payoff functions $v^{m}$ and $u_{n}$ are continuous on the compact set of outcomes $\mathcal{X}$ for all $(m, n) \in \mathcal{M} \times \mathcal{N}$.

By the Weierstrass theorem (Bertsekas 1995, p. 540) any continuous function on a compact set has a nonempty set of maximizers; by Berge's (1963, p. 116) maximum theorem this set is also compact. When defining the contracting game $\Gamma$ in (3) we restrict the choice of the principals' transfer schedules to the set of continuous functions. Together with Assumption 1 this restriction ensures that all participants' net payoffs are continuous and therefore that given any transfer schedule $t$ and given all other agents' actions, an optimal action $x_{n}$ in agent $n$ 's second-stage game (described by (4)) exists. Still, if agents' net payoffs are nonconcave it may be the case that their set of maximizers becomes nonconvex, so that the existence of an equilibrium is not implied by Kakutani's fixed-point theorem.

Let us now consider the question of the existence of a WTE that implements an efficient outcome using general (continuous) outcome-contingent transfer schedules. It turns out that, as long as all participants' payoffs are continuous, our solution to the contracting game (cf. the next section) resolves this question of existence in a positive, constructive and thus quite satisfying way.

If transfer schedules are restricted to be action-contingent, i.e., any contractual payment from a principal to an agent can depend only on that agent's action, then the existence of an efficient WTE cannot be guaranteed. In Section 4 we provide one example where coordination of a supply chain with action-contingent contracts is not possible, while it can naturally be coordinated using

\footnotetext{
${ }^{10}$ We use the following definition of (uniform) continuity on the compact set $\mathcal{X} \subset \mathbb{R}_{+}^{M N L}:$ a function $g: \mathcal{X} \rightarrow \mathbb{R}$ is continuous on $\mathcal{X}$ if, given any $x \in \mathcal{X}$, for any $\varepsilon>0$ there exists a $\delta=\delta(\varepsilon)>0$ such that $\hat{x} \in\{\xi \in \mathcal{X}:\|\xi-x\|<$ $\delta\} \Rightarrow|g(\hat{x})-g(x)|<\varepsilon$, where $\|\cdot\|$ is a given norm on the Euclidean space $\mathbb{R}^{M N L}$. Note that according to this definition any function is continuous whenever $\mathcal{X}$ is finite.
} 
outcome-contingent contracts. ${ }^{11}$ In the absence of externalities in the agents' payoff functions any WTE $(\hat{t}, \hat{x})$ is efficient (cf. P\&R) and equilibrium transfer schedules are action-contingent. Indeed, the equilibrium conditions (WT) and (AM) then immediately imply (6). However, this result breaks down in the presence of payoff externalities: by Lemma 1 an efficient WTE with action-contingent contracts does not exist whenever it is impossible to find a transfer $\hat{t}$ for which the resulting fixed-point problem (PM) has an efficient solution $\hat{x}$ that also satisfies (7). The reason for this is that for any given transfer schedule the set of outcomes $x^{\circ} \in \mathcal{X}$ for which (7) holds might not contain an efficient outcome that also satisfies (PM) for that transfer schedule. ${ }^{12}$

Since we are interested in the existence of an efficient WTE, let $\hat{x}$ be any efficient outcome. The following two assumptions are helpful in establishing the existence of a WTE $(\hat{t}, \hat{x})$ with actioncontingent transfer schedules in Section 3.3; they are not needed for the rest of our results.

Assumption 2 (Payoff Concavity) Given an efficient outcome $\hat{x} \in \mathcal{X}$, principal m's gross payoff $v^{m}(\cdot)$ and agent $n$ 's gross payoff $u_{n}\left(\cdot, \hat{x}_{-n}\right)$ are concave for all $(m, n) \in \mathcal{M} \times \mathcal{N}$.

Assumption 3 (Action-Set Convexity) The agents' action sets $\mathcal{X}_{1}, \ldots, \mathcal{X}_{N}$ are convex.

Under Assumption 2 and Assumption 3 P\&R (Theorem 8) guarantee the existence of an efficient WTE (in the absence of payoff externalities). We emphasize that their existence proof, based on a Banach-space generalization of Farkas' Lemma (Aubin and Ekeland 1984, p. 144), is highly nonconstructive and therefore offers no insights as to how to actually design efficient contracts, the question of central practical importance. In Section 3 we provide a simple explicit equilibrium construction (in the presence of payoff externalities) and thus resolve the question of the existence of action-contingent equilibrium contracts in a satisfying and direct way. In the presence of externalities Assumption 2 requires only the agents' payoff concavity with respect to their own actions, not all other agents' actions. We also note that the payoff concavity and action-set convexity, although sufficient, are not at all necessary for the existence of an efficient WTE.

\subsection{Standard Two-Echelon Supply-Chain Modelling}

At the end of Section 2.1 we pointed out that our model directly applies to bottom-up contracting and needs some slight modifications to accommodate top-down contracting. The reason for these modifications is that our model assumes that the transfers from principals to agents are nonnegative (since $\mathcal{T} \subseteq \mathbb{R}_{+}^{M L}$ ), so that the agents' participation is guaranteed to be individually rational. It is fairly straightforward to transform a top-down contracting situation (with arbitrary bounded transfers from agents to principals) into our standard bottom-up contracting framework (with nonnegative transfers from principals to agents) by interpreting outcome-contingent variations of a transfer schedule from an agent to a principal as a nonnegative variable discount that the principal

\footnotetext{
${ }^{11}$ We are grateful to Hau Lee for his suggestion to distinguish action contingency and outcome contingency.

${ }^{12}$ In a simple model with one principal and multiple agents Segal (1999) shows that in the presence of payoff externalities an efficient outcome may not be implemented as a consequence. His necessary and sufficient condition for implementability (condition (5), ibid., p. 354) corresponds to our condition (7) in Definition 2.
} 
offers to the agent, off a sufficiently high outcome-contingent 'base transfer schedule' from that the agent to the principal.

Consider a two-echelon supply chain with suppliers (upstream firms) as principals and the retailers (downstream firms) as agents. Each seller offers a menu of contracts to each buyer. In a top-down contracting game the transfer payment goes from the buyers to the sellers, i.e., from the agents to the principals. We now modify the payoffs as follows. First, we choose for each agent $n$ an arbitrary reference point $\tilde{x}_{n}=\left(\tilde{x}_{n}^{1}, \ldots, \tilde{x}_{n}^{M}\right) \in \mathcal{X}_{n}$ and let

$$
\tilde{u}_{n}(x)=u_{n}(x)-\sum_{m \in \mathcal{M}} w_{n}^{m} \Lambda_{n}^{m}\left(x_{n}^{m}-\tilde{x}_{n}^{m}\right),
$$

where $w_{n}^{m}$ are appropriate nonnegative constants and each $\Lambda_{n}^{m} \in C\left(\mathbb{R}_{+}^{L}, \mathbb{R}\right)$ is convex and such that

$$
\left\{\left(x_{n}^{m}, x_{n}^{-m}\right) \in \mathcal{X}_{n}: \Lambda_{n}^{m}\left(x_{n}^{m}-\tilde{x}_{n}^{m}\right) \geq 0\right\}=\left\{\tilde{x}_{n}\right\} .
$$

The functions $\Lambda_{n}^{m}$ can be seen as functions that penalize deviations from the reference point. The constants $w_{n}^{m}$ and functions $\Lambda_{n}^{m}$ are chosen such that the slope around the reference point is large enough (in absolute value) so that

$$
\tilde{x}_{n}=\arg \max _{x_{n} \in \mathcal{X}_{n}} \tilde{u}_{n}\left(x_{n}\right) .
$$

In other words, given no transfer from any principal, agent $n$ chooses $\tilde{x}_{n}$ as his strictly preferred action. ${ }^{13}$ Defining principal $m$ 's modified payoff by

$$
\tilde{v}^{m}(x)=v^{m}(x)+\sum_{n \in \mathcal{N}} w_{n}^{m} \Lambda_{n}^{m}\left(x_{n}^{m}-\tilde{x}_{n}^{m}\right)
$$

ensures strategic equivalence of the game $\tilde{\Gamma}$ in the modified payoffs with the original game $\Gamma$. Indeed, note first that any efficient outcome $\hat{x}$ of $\Gamma$ is also an efficient outcome of $\tilde{\Gamma}$ and vice-versa, since total surplus $W$ remains unaffected by the changes. In addition, note that in the game with modified payoffs each agent $n$ requires a strictly positive transfer payment to implement any action other than $\tilde{x}_{n}$. Relation (8) together with the convexity of $\Lambda_{n}^{m}$ implies that $\Lambda_{n}^{m}\left(x_{n}^{m}\right)$ is nonnegative for all feasible $x_{n}^{m}$. If we then consider the modified transfer

$$
\tilde{t}_{n}^{m}(x)=t_{n}^{m}(x)+w_{n}^{m} \Lambda_{n}^{m}\left(x_{n}^{m}-\tilde{x}_{n}^{m}\right) \geq 0,
$$

which is admissible (i.e., nonnegative) if $w_{n}^{m}$ is large enough, it is clear that the principals' and the agents' net payoffs are identical in $\tilde{\Gamma}$ and $\Gamma$. Note that for $w_{n}^{m}$ large enough, despite the possible negativity of $t_{n}^{m}$ in the top-down contracting setting, the modified transfer $\tilde{t}_{n}^{m}$ is always nonnegative (and thus admissible) in equilibrium.

Remark 1 (Wholesale Discounts) If $\mathcal{X}_{n}=\mathbb{R}_{+}^{M L}$, then with $\tilde{x}=0$ it is possible to choose $\Lambda_{n}^{m}\left(x_{n}^{m}-\tilde{x}_{n}^{m}\right)=x_{n}^{m}$. The resulting linearly augmented modified transfer

$$
\tilde{t}_{n}^{m}(x)=t_{n}^{m}(x)+w_{n}^{m} x_{n}^{m}
$$

\footnotetext{
${ }^{13}$ For instance, if $\tilde{x}_{n}^{m}$ is an interior local maximizer of $u_{n}$ on $\mathcal{X}_{n}$, then one might choose $\Lambda_{n}^{m}\left(x_{n}^{m}-\tilde{x}_{n}^{m}\right)=\left\|x_{n}^{m}-\tilde{x}_{n}^{m}\right\|_{2}^{2}$. In that case, if in addition the maximizer $\tilde{x}_{n}$ is global on $\mathcal{X}_{n}$, then any positive constant $w_{n}^{m}>0$ ensures that the maximizer becomes strict.
} 
can for a given $x_{-n}$ be interpreted as (negative) quantity discounts $t_{n}^{m}\left(x_{n}, x_{-n}\right)$ being offered by principal $m$ to agent $n$ relative to a sufficiently "expensive" wholesale price $w_{n}^{m}$.

REMARK 2 (Missing Links) If there is no interaction between principal $m$ and agent $n$ in the two-tier supply chain (e.g., due to exogenous trade restrictions), i.e., when $\mathcal{T}_{n}^{m}=\{0\}$, then agent $n$ chooses his action $x_{n}$ independently of principal $m$. More specifically, he selects an action $x_{n}=$ $\left(\hat{x}_{n}^{m}, x_{n}^{-m}\right)$ that maximizes his own payoffs, given the proposed transfers by principals with trading links to him. As a result, any efficient outcome $\hat{x}$ contains the component $\hat{x}_{n}^{m}\left(\hat{x}_{n}^{-m}\right)$ so chosen; moreover, by principal $m$ 's cost minimization (i.e., condition (PM)) her transfer $\hat{t}_{n}^{m}$ will vanish in equilibrium. We therefore do not have to pay special attention to missing trading links, as they can be accommodated quite naturally in our framework.

\section{Efficient Contract Design}

In this section we study how efficient outcomes can be implemented in a supply chain or other setting by an appropriate contract design. Our approach is nonparametric and we are looking for a set of (nonlinear) transfers $\hat{t}=\left[\hat{t}_{n}^{m}\right]$ that implement $(\hat{t}, \hat{x})$ as a WTE. To accomplish this, it is useful first to simplify the problem of solving the system (WT),(AM),(PM), which leads to a much simpler "reduced contract-design problem" based on (WT) and (AM). Any solution to the latter problem can then be mapped to a solution of the original problem by adding appropriate constant transfers, such that the resulting equilibrium payment schedules satisfy the nonnegativity constraint as well as (PM). In all of this section we maintain Assumption 1 (payoff continuity). With the exception of the results on action-contingent contracts in Section 3.3, payoff continuity is all that is needed to provide a constructive solution to the general contract-design problem with payoff externalities.

\subsection{The Reduced Contract-Design Problem}

Based on the characterization of weakly truthful equilibria in Theorem 1, we now turn to the practical problem of designing contracts that implement an efficient outcome. ${ }^{14}$ For this we consider a reduced contract-design problem first, the solution to which can be directly mapped to a solution of the original efficient contract-design problem. Given an efficient outcome $\hat{x} \in \mathcal{X}$, let

$$
F^{m}(x)=v^{m}(x)-v^{m}(\hat{x})
$$

denote principal $m$ 's excess revenue from implementing $x$ instead of $\hat{x}$, and let

$$
G_{n}(x)=u_{n}(\hat{x})-u_{n}(x)
$$

denote agent $n$ 's excess cost from taking an action $x_{n}$ instead of $\hat{x}_{n}$ given the other agents' actions $\hat{x}_{-n}$. If $t_{n}^{m}\left(x_{n}\right)$ represents a direct transfer from principal $m$ to agent $n$ contingent on his taking action $x_{n} \in \mathcal{X}_{n}$, then it is useful to consider the excess transfer,

$$
\Delta_{n}^{m}(x)=t_{n}^{m}(x)-t_{n}^{m}(\hat{x}),
$$

\footnotetext{
${ }^{14}$ Any such outcome can be determined as a maximizer of $W$ on $\mathcal{X}$ (cf. Definition 2).
} 
relative to agent $n$ 's efficient action $\hat{x}_{n}$. Thus, by only looking at deviations from the efficient outcome $\hat{x}$ we can rewrite conditions (WT) and (AM) equivalently in terms of excess measures, which yields

$$
F^{m}(x) \leq \sum_{n \in \mathcal{N}} \Delta_{n}^{m}(x)
$$

for any principal $m \in \mathcal{M}$, and

$$
G_{n}\left(x_{n}, \hat{x}_{-n}\right) \geq \sum_{m \in \mathcal{M}} \Delta_{n}^{m}\left(x_{n}, \hat{x}_{-n}\right)
$$

for any agent $n \in \mathcal{N}$. In other words, at any efficient equilibrium of the underlying game $\Gamma$ there does not exist a more profitable outcome than $\hat{x}$ for any principal, and any agent's excess cost of implementing any action different from $\hat{x}_{n}$ outweighs the excess transfer he could obtain, which thus keeps him from deviating. To obtain a solution to the system of inequalities (WT'),(AM') it is useful to find a matrix function

$$
\Delta=\left[\Delta_{n}^{m}\right] \in C\left(\mathcal{X}, \mathbb{R}^{M \times N}\right)
$$

which solves the following stronger reduced contract-design problem (RCDP),

$$
F^{m}(x)-\sum_{j \in \mathcal{N}} \Delta_{j}^{m}(x) \leq 0 \leq G_{n}(x)-\sum_{i \in \mathcal{M}} \Delta_{n}^{i}(x),
$$

for all $(m, n) \in \mathcal{M} \times \mathcal{N}$ and all $x \in \mathcal{X}$. The first inequality in (R) is equivalent to (WT') while the second inequality in (R) implies (AM') for any given outcome $\hat{x} \in \mathcal{X}$. The following result describes a class of solutions up to a constant matrix (at $\hat{x}$ ), so that without loss of generality it is possible to restrict attention to solutions of the RCDP that also satisfy $\Delta(\hat{x})=0$, a necessary condition for representing excess transfers in the form (11).

Lemma 2 (i) Any solution $\Delta$ to the reduced contract-design problem (R) satisfies

$$
\sum_{i \in \mathcal{M}} \Delta_{n}^{i}(\hat{x})=\sum_{j \in \mathcal{N}} \Delta_{j}^{m}(\hat{x})=0
$$

for all $(m, n) \in \mathcal{M} \times \mathcal{N}$. (ii) If $\Delta$ is a solution to $(R)$ and $\delta \in \mathbb{R}^{M \times N}$ is a constant matrix, then $\Delta+\delta$ also solves the reduced contract-design problem if and only if

$$
\sum_{i \in \mathcal{M}} \delta_{n}^{i}=\sum_{j \in \mathcal{N}} \delta_{j}^{m}=0
$$

for all $(m, n) \in \mathcal{M} \times \mathcal{N}$.

Lemma 2 implies that the excess transfer $\bar{\Delta}(x)=\Delta(x)-\Delta(\hat{x})$ (satisfying $\bar{\Delta}(\hat{x})=0$ ) is a solution to the reduced contract-design problem, if only $\Delta$ solves the system of inequalities (R). ${ }^{15}$ Thus, in everything that follows we consider only solutions to the reduced contract-design problem that satisfy $\Delta(\hat{x})=0$ without having to impose this condition as an extra constraint in the search for a

\footnotetext{
${ }^{15}$ Note that $\delta=-\Delta(\hat{x})$ satisfies (13) as a direct consequence of (12).
} 
solution. If such an excess-transfer matrix $\Delta$ has been found, then as a consequence of Theorem 1 the function

$$
t_{n}^{m}\left(x_{n}\right)=\Delta_{n}^{m}\left(x_{n}\right)+\alpha_{n}^{m},
$$

with $\alpha_{n}^{m} \in \mathbb{R}_{+}$some appropriate nonnegative constant, is a candidate equilibrium transfer from principal $m$ to agent $n$. The constant $\alpha_{n}^{m}$ thereby corresponds to the payment contingent on the equilibrium outcome, i.e., $t_{n}^{m}\left(\hat{x}_{n}\right)=\alpha_{n}^{m}$.

In addition to the reduction of the solution space to solutions that vanish at the efficient outcome we also obtain that the set of solutions to the reduced contract-design problem is convex.

Lemma 3 Given any two solutions $\Delta, \tilde{\Delta}$ to the reduced contract-design problem $(R)$, the convex combination $\lambda \Delta+(1-\lambda) \tilde{\Delta}$ is also a solution, for any $\lambda \in(0,1)$.

The preceding results provide some properties of the class of solutions to the RCDP. Clearly, this class can be expected to contain more than one solution, since the $N+M$ inequalities in (R) are not enough to pin down an $N \times M$ solution matrix $\Delta$. To obtain a set of solutions to (R) it is in fact fully sufficient to specify only the row sums $R^{m}=\sum_{j \in \mathcal{N}} \Delta_{j}^{m}$ and column sums $C_{n}=\sum_{i \in \mathcal{M}} \Delta_{n}^{i}$ that solve the system

$$
\left\{\begin{array}{l}
F^{m}(x)-R^{m}(x) \leq 0 \leq G_{n}(x)-C_{n}(x), \\
\sum_{i \in \mathcal{M}} R^{i}=\sum_{j \in \mathcal{N}} C_{j},
\end{array}\right.
$$

for all $(m, n) \in \mathcal{M} \times \mathcal{N}$, which is equivalent to $(\mathrm{R})$. We now provide an explicit solution to ( $\left.\mathrm{R}^{\prime}\right)$.

Theorem 2 (Solution to the Reduced Contract-Design Problem) Let $\hat{x} \in \mathcal{X}$ be an efficient outcome. The functions $\left(R^{1}, \ldots, R^{M}\right),\left(C_{1}, \ldots, C_{N}\right)$ with $R^{m}, C_{n} \in C(\mathcal{X}, \mathbb{R})$ constitute a solution to $\left(R^{\prime}\right)$ if and only if

$$
\left\{\begin{aligned}
R^{m}\left(x ; f^{m}\right) & =F^{m}(x)+f^{m}(x), \\
C_{n}\left(x ; g_{n}\right) & =G_{n}(x)-g_{n}(x),
\end{aligned}\right.
$$

for all $(m, n) \in \mathcal{M} \times \mathcal{N}$ and all $x \in \mathcal{X}$, where the functions $f^{m}, g_{n} \in C\left(\mathcal{X}, \mathbb{R}_{+}\right)$are such that $f^{m}(\hat{x})=$ $g_{n}(\hat{x})=0$ and

$$
\sum_{i \in \mathcal{M}} f^{i}(x)+\sum_{j \in \mathcal{N}} g_{j}(x)=W(\hat{x})-W(x)
$$

on $\mathcal{X}$.

Corollary 1 (Existence) (i) A continuous solution to ( $\left.R^{\prime}\right)$ exists. (ii) The functions $\left(R^{1}, \ldots, R^{M}\right)$, $\left(C_{1}, \ldots, C_{N}\right)$ with

$$
\left\{\begin{aligned}
R^{m}(x) & =F^{m}(x)+\varphi^{m}(x)(W(\hat{x})-W(x)), \\
C_{n}(x) & =G_{n}(x)-\gamma_{n}(x)(W(\hat{x})-W(x)),
\end{aligned}\right.
$$

for all $(m, n) \in \mathcal{M} \times \mathcal{N}$ and all $x \in \mathcal{X}$ constitute a solution to ( $\left.R^{\prime}\right)$ as long as the functions $\varphi^{m}, \gamma_{n} \in$ $C\left(\mathcal{X}, \mathbb{R}_{+}\right)$are such that $\sum_{i \in \mathcal{M}} \varphi^{i}+\sum_{j \in \mathcal{N}} \gamma_{j}=1$. 
Proof. (ii) By setting $f^{m}(x) \equiv \varphi^{m}(x)(W(\hat{x})-W(x)) \geq 0$ and $g_{n}(x) \equiv \gamma_{n}(x)(W(\hat{x})-W(x)) \geq 0$ on $\mathcal{X}$ we obtain, by applying Theorem 2 , that the functions $\left(R^{1}, \ldots, R^{M}\right),\left(C_{1}, \ldots, C_{n}\right)$ constitute a solution to (R'). (i) The existence of a solution to (R') follows immediately by setting $\varphi^{m}=1 / M$ and $\gamma_{n}=0$ for all $(m, n) \in \mathcal{M} \times \mathcal{N}$.

Note that the solution to ( $\mathrm{R}$ ') exists, independently of any assumptions about the participants' payoff functions. It depends solely on the existence of an efficient outcome $\hat{x} \in \mathcal{X}$. The solution (14) is such that the sum of the principals' excess transfers to a given agent $n$ at least compensates for that agent's excess cost $G_{n}(x)$ of implementing the efficient outcome $\hat{x}$ instead of $x$. Similarly, the sum of all excess transfers that originate from a given principal $m$ needs to (at least weakly) exceed that principal's excess payoff $F^{m}(x)$ from implementing the outcome $x$ instead of the efficient outcome $\hat{x}$. Corollary 1 shows these requirements can be met by simply allocating a fraction of the nonnegative surplus difference $W(\hat{x})-W(x)$ to each principal and each agent. Corollary 2 shows that all solutions to the reduced contract-design problem $(\mathrm{R})$ can be characterized in terms of solutions to ( $\left.\mathrm{R}^{\prime}\right)$.

Corollary 2 (Characterization) The excess-transfer matrix $\Delta$ solves the reduced contract-design problem $(R)$ if and only if it is such that

$$
\left\{\begin{array}{c}
\Delta_{n}^{M}=C_{n}-\sum_{i=1}^{M-1} \Delta_{n}^{i}, \\
\Delta_{N}^{m}=R^{m}-\sum_{j=1}^{N-1} \Delta_{j}^{m},
\end{array}\right.
$$

for all $(m, n) \in \mathcal{M} \times \mathcal{N}$, where $\Delta_{-N}^{-M}=\left[\Delta_{j}^{i}\right]_{i, j=1}^{M-1, N-1}$ is any continuous excess-transfer matrix with $\Delta_{-N}^{-M}(\hat{x})=0$, and $\left(R^{1}, \ldots, R^{M}\right),\left(C_{1}, \ldots, C_{N}\right)$ is a solution to $\left(R^{\prime}\right)$.

Proof. From (17) it is evident that $\sum_{i \in \mathcal{M}} \Delta_{n}^{i}=C_{n}$ and $\sum_{j \in \mathcal{N}} \Delta_{j}^{m}=R^{m}$ for all $(m, n) \in$ $\{1, \ldots, M-1\} \times\{1, \ldots, N-1\}$. Consider now $\Delta_{N}^{M}=C_{N}-\sum_{i=1}^{M-1} \Delta_{N}^{i}=C_{N}-\sum_{i=1}^{M-1}\left(R^{i}-\sum_{j=1}^{N-1} \Delta_{j}^{i}\right)$. If we set $R=\sum_{m \in \mathcal{M}} R^{m}$ and $C=\sum_{n \in \mathcal{N}} C_{n}$, then by (R') it is $R=C$ and thus $\Delta_{N}^{M}=C_{N}+$ $R^{M}-R+\sum_{i, j=1}^{M-1, N-1} \Delta_{j}^{i}=C_{N}+R^{M}-C+\sum_{i, j=1}^{M-1, N-1} \Delta_{j}^{i}$. In other words, both equations in (17) provide the same specification for $\Delta_{N}^{M}$. In addition, it is $\sum_{i \in \mathcal{M}} \Delta_{N}^{i}=C_{N}$ and $\sum_{j \in \mathcal{N}} \Delta_{j}^{m}=R^{M}$, so that (17) indeed describes a solution to the reduced contract-design problem (R).

Note that by re-indexing the principals and agents one can replace $\Delta_{-N}^{-M}$ in Corollary 2 by $\Delta_{-l}^{-k}$ for any given $(k, l) \in \mathcal{M} \times \mathcal{N}$. That means that one can use any principal and any agent as residual recipient (or "buffer") for excess payoffs, provided that excess transfers for the other principals and agents have been fixed. We emphasize the fundamental flexibility the characterization of the solution to the RCDP in (17) provides. By leaving the excess transfers of $\Delta_{n}^{m}$ for $(m, n) \in$ $\{1, \ldots, M-1\} \times\{1, \ldots, N-1\}$ free for design, it is possible to coordinate a multi-principal multiagent supply chain using many different transfer schemes (including revenue sharing, cost sharing, or surplus sharing) for almost all principals and almost all agents. The contract-design example in Section 4.1 helps to clarify this point. 
Symmetric Solutions. From our preceding discussion it becomes clear that the buffer principal and/or the buffer agent generally bear the asymmetries resulting from particular design choices. It is therefore natural to ask under what conditions coordinating contracts (in terms of solutions to the RCDP) can be symmetric across the supply chain, and one naturally obtains the existence of a symmetric solution, if the payoff structure is symmetric in a general sense. We say that the contract design game $\Gamma$ in (3) has a symmetric payoff structure if (i) $\mathcal{X}_{1}=\cdots=\mathcal{X}_{N}$, and (ii) there exist gross payoff functions $u, v \in C(\mathcal{X}, \mathbb{R})$ such that

$$
\left\{\begin{aligned}
v^{m}\left(x^{m}, x^{-m}\right) & =v\left(\pi\left(x_{1}^{m}, \ldots, x_{N}^{m}\right), \pi\left(x^{-m}\right)\right), \\
u_{n}\left(x_{n}, x_{-n}\right) & =u\left(\pi\left(x_{n}^{1}, \ldots, x_{n}^{M}\right), \pi\left(x_{-n}\right)\right)
\end{aligned}\right.
$$

for all $(m, n) \in \mathcal{M} \times \mathcal{N}$, where $\pi(x)$ denotes a permutation of (possibly vector-valued) elements of a vector $x=\left(x_{1}^{1}, \ldots, x_{N}^{1} ; x_{1}^{2}, \ldots, x_{N}^{2} ; \ldots ; x_{1}^{M}, \ldots, x_{N}^{M}\right) .{ }^{16}$ In other words, if the contracting game has a symmetric payoff structure, then by (18) the principals care about outcomes but not who exactly implements them. In addition, any agent cares about his own action and the other agents' actions, but is indifferent about changing the labels of the other agents.

Corollary 3 (Symmetric Solution) If the contract design game $\Gamma$ has a symmetric payoff structure, then the excess-transfer matrix $\Delta=\left[\Delta_{n}^{m}\right]$ with

$$
\Delta_{n}^{m}(x ; \lambda)=\frac{1-\lambda}{M}\left(u(\hat{x})-u\left(x_{n}, x_{-n}\right)\right)+\frac{\lambda}{N}\left(v\left(x^{m}, x^{-m}\right)-v(\hat{x})\right)
$$

for all $(m, n) \in \mathcal{M} \times \mathcal{N}$, all $x \in \mathcal{X}$, and some $\lambda \in[0,1]$ solves the reduced contract-design problem (R), as long as

$$
\frac{1-\lambda}{M}(\bar{\mu}-\hat{w}) \leq 0 \leq \frac{\lambda}{N}(\hat{w}-\underline{\mu})
$$

where $\hat{w} \equiv M v(\hat{x})+N u(\hat{x})$ denotes the total welfare at the efficient outcome, and ${ }^{17}$

$$
\underline{\mu} \equiv \max _{x \in \mathcal{X}}\left\{\sum_{i \in \mathcal{M}} v\left(x^{i}, x^{-i}\right)+N u\left(x_{n}, x_{-n}\right)\right\}, \quad \bar{\mu} \equiv \max _{x \in \mathcal{X}}\left\{\sum_{j \in \mathcal{N}} u\left(x_{j}, x_{-j}\right)+M v\left(x^{m}, x^{-m}\right)\right\} .
$$

Proof. Instead of relying on the characterization of solutions to the equivalent reformulation (R') of the RCDP, it is more convenient to verify directly that the reduced transfers defined by (19) satisfy (R). Using (18) and (20) we have that

$$
\begin{aligned}
G_{n}\left(x_{n}, x_{-n}\right)-\sum_{i \in \mathcal{M}} \Delta_{n}^{i}\left(x_{n}, x_{-n}\right) & =\frac{\lambda \hat{w}}{N}-\lambda u\left(x_{n}, x_{-n}\right)-\frac{\lambda}{N} \sum_{i \in \mathcal{M}} v\left(\left(x_{n}^{i}, x_{-n}^{i}\right),\left(x_{n}^{-i}, x_{-n}^{-i}\right)\right) \\
& =\frac{\lambda}{N}\left(\hat{w}-N u\left(x_{n}, x_{-n}\right)-\sum_{i \in \mathcal{M}} v\left(x^{i}, x^{-i}\right)\right) \geq \frac{\lambda}{N}(\hat{w}-\underline{\mu}) \\
& \geq 0
\end{aligned}
$$

\footnotetext{
${ }^{16}$ The $L$-dimensional elements $x_{j}^{i}$ remain unaffected by the permutation.

${ }^{17}$ The constants $\underline{\mu}$ and $\bar{\mu}$ are well defined (for the maxima do indeed exist) and are independent of $n$ and $m$ respectively (for the payoff structure is by assumption symmetric).
} 
for all $n \in \mathcal{N}$ and all $x \in \mathcal{X}$. Similarly, from (18) and (20) we also obtain that

$$
\begin{aligned}
F^{m}\left(x^{m}, x^{-m}\right)-\sum_{j \in \mathcal{N}} \Delta_{j}^{m}\left(x^{m}, x^{-m}\right) & =\frac{1-\lambda}{M}\left(\sum_{j \in \mathcal{N}} u\left(\left(x_{j}^{m}, x_{j}^{-m}\right),\left(x_{-j}^{m}, x_{-j}^{-m}\right)\right)+M v\left(x^{m}, x^{-m}\right)-\hat{w}\right) \\
& =\frac{1-\lambda}{M}\left(\sum_{j \in \mathcal{N}} u\left(x_{j}, x_{-j}\right)+M v\left(x^{m}, x^{-m}\right)-\hat{w}\right) \leq \frac{1-\lambda}{M}(\bar{\mu}-\hat{w}) \\
& \leq 0
\end{aligned}
$$

for all $m \in \mathcal{M}$ and all $x \in \mathcal{X}$, which completes our proof.

A symmetric solution is extremely useful for examining the comparative statics of complex supply chains with respect to a change in the number of principals and/or the number of agents. The symmetric solution is also desirable in situations which exhibit a symmetric payoff structure. However, an examination of the proof to Corollary 3 shows that, in order for a symmetric solution of the form (19) to exist, it is necessary that relation (20) hold for at least one $\lambda \in[0,1]$. The additional condition requires that, first, any principal's payoffs plus the average of the agents' payoffs shall not exceed the average of the system surplus split evenly among principals, and, second, that any agent's payoffs plus the average of the principals' payoffs shall not exceed the average of the system surplus split among the agents. In other words, to interpret condition (20), replicating any single agent or principal while shutting all other not replicated parties down does not make it possible to increase surplus beyond the efficient system surplus $\hat{w}$.

\subsection{Solving the General Contract-Design Problem}

We are now ready to state the key result for the design of efficient multi-principal multi-agent contracts, given any solution to the reduced contract-design problem.

Theorem 3 (Efficient Contract Design) If the excess-transfer matrix $\Delta=\left[\Delta_{n}^{m}\right]$ solves the reduced contract-design problem $(R)$ implementing the efficient outcome $\hat{x}$, then $(\hat{t}, \hat{x})$ with

$$
\hat{t}_{n}^{m}\left(x ; \vartheta_{n}^{m}, \theta_{n}\right)=\hat{\Delta}_{n}^{m}\left(x ; \vartheta_{n}^{m}, \theta_{n}\right)-\min _{x_{n} \in \mathcal{X}_{n}}\left\{\hat{\Delta}_{n}^{m}\left(x_{n}, \hat{x}_{-n} ; \vartheta_{n}^{m}+\theta_{n}, \vartheta_{n}^{m} \theta_{n} /\left(\vartheta_{n}^{m}+\theta_{n}\right)\right)\right\}
$$

and

$$
\hat{\Delta}_{n}^{m}\left(x ; \vartheta_{n}^{m}, \theta_{n}\right)=\Delta_{n}^{m}(x)+\vartheta_{n}^{m}(x)\left(1-\theta_{n}(x)\right)\left(G_{n}(x)-\sum_{i \in \mathcal{M}} \Delta_{n}^{i}(x)\right)
$$

for all $(m, n) \in \mathcal{M} \times \mathcal{N}, \theta_{n} \in C\left(\mathcal{X}_{n},\left[0, \bar{\theta}_{n}\right]\right)$ with $\bar{\theta}_{n}=\sup \left\{\hat{\theta}_{n} \in[0,1]: \min _{i \in \mathcal{M}} \hat{t}_{n}^{i}\left(\hat{x} ; \vartheta_{n}^{i}, \hat{\theta}_{n}\right) \geq 0\right\}$ and arbitrary $\vartheta_{n}^{m} \in C\left(\mathcal{X}_{n},[0,1]\right)$ satisfying $\sum_{i \in \mathcal{M}} \vartheta_{n}^{i}(x) \equiv 1$, is a WTE of $\Gamma$.

The last result is essential for specifying coordinating contracts implementing a given efficient outcome $\hat{x}$ : any solution $\Delta$ of the RCDP can be mapped to a solution of the original contract-design problem (WT),(AM),(PM) by the outcome-contingent transformation (21)-(22). The modified 
transfer matrix $\hat{\Delta}=\left[\hat{\Delta}_{n}^{m}\right]$ also satisfies the reduced contract-design problem $(\mathrm{R})$. The constant nonnegative shifts

$$
\alpha_{n}^{m}\left(\vartheta_{n}^{m}, \theta_{n}\right)=-\min _{x_{n} \in \mathcal{X}_{n}}\left\{\hat{\Delta}_{n}^{m}\left(x_{n}, \hat{x}_{-n} ; \vartheta_{n}^{m}+\theta_{n}, \vartheta_{n}^{m} \theta_{n} /\left(\vartheta_{n}^{m}+\theta_{n}\right)\right)\right\} \geq 0
$$

of the modified excess transfers $\hat{\Delta}_{n}^{m}$ correspond exactly to the amounts transferred from principal $m$ to agent $n$ in equilibrium. These amounts generally depend on the outcome-contingent convex combination selected in (22). Generally, for each agent $n \in \mathcal{N}$ the principals are in a conflict about who should pay him less, since the higher $\vartheta_{n}^{m}$, the lower principal $m$ 's transfer to agent $n$ in equilibrium. If for principal $m$ the weight $\vartheta_{n}^{m}=1$, her equilibrium transfer to agent $n$ is indeed as small as possible, given the solution $\Delta$ to the reduced contract-design problem $(\mathrm{R})$. This conflict of interest is not resolved as part of the game; it is a consequence of the multiplicity of coordinating equilibria. The multiplicity can be resolved using pre-play communication including a cooperative bargaining procedure, which is beyond the scope of this paper. The function $\theta_{n}$ can be interpreted as a perturbation of the contracts which reflects the "strength" of the implementation from the agents' point of view. For $\theta_{n}=0$, which is always feasible, the implementation of the efficient outcome is weak in the sense that all agents are indifferent between implementing any action $x_{n} \in \mathcal{X}_{n}{ }^{18}$ Such a weak implementation of the coordinating outcome is not robust and thus not particularly desirable. The implementation can be strengthened for agent $n$ if $\bar{\theta}_{n}>0$.

Corollary 4 (Strong Implementation) If in addition to the assumptions in Theorem 3 we have that (i) there is only one efficient outcome $\hat{x}$ in $\mathcal{X}$, and (ii) $\bar{\theta}_{n}>0$, then there exists a transfer matrix $\hat{t}=\left[\hat{t}_{n}^{m}\right]$ such that $(\hat{t}, \hat{x})$ is a WTE, and

$$
u_{n}(\hat{x})+\sum_{i \in \mathcal{M}} \hat{t}_{n}^{i}(\hat{x})>u_{n}\left(x_{n}, \hat{x}_{-n}\right)+\sum_{i \in \mathcal{M}} \hat{t}_{n}^{i}\left(x_{n}, \hat{x}_{-n}\right)
$$

for all $x_{n} \in \mathcal{X}_{n} \backslash\left\{\hat{x}_{n}\right\}$.

Proof. Since by assumption $W(\hat{x})>W(x)$ for all $x \in \mathcal{X} \backslash\{\hat{x}\}$, we have by Corollary 1 that the functions $\left(R^{1}, \ldots, R^{M}\right),\left(C_{1}, \ldots, C_{N}\right)$ with $R^{m}(x)=F^{m}(x)$ and $C_{n}(x)=G_{n}(x)-(W(\hat{x})-$ $W(x)) / N<G_{n}(x)$ for all $(m, n) \in \mathcal{M} \times \mathcal{N}$ and all $x \in \mathcal{X} \backslash\{\hat{x}\}$ constitute a solution to (R'). If we set $\vartheta_{n}^{m}=1 / M$ and $\theta_{n}=\bar{\theta}_{n} / 2>0$, then

$$
G_{n}\left(x_{n}, \hat{x}_{n}\right)-\sum_{i \in \mathcal{M}} \hat{\Delta}_{n}^{i}\left(x_{n}, \hat{x}_{n} ; \vartheta_{n}^{i}, \theta_{n}\right)=\frac{\bar{\theta}_{n}}{2}\left(G_{n}\left(x_{n}, \hat{x}_{-n}\right)-C_{n}\left(x_{n}, \hat{x}_{-n}\right)\right)>0
$$

for all $x_{n} \in \mathcal{X}_{n} \backslash\left\{\hat{x}_{n}\right\}$, which (using the equivalence of (AM') with (AM)) completes our proof.

The general result in Corollary 4 is of significant practical importance: as long as the efficient outcome $\hat{x}$ is unique and the equilibrium transfer to an agent $n$ is nonzero (which implies that $\bar{\theta}_{n}>$ $0)$, it is possible to implement the efficient outcome strongly in the sense that agent $n$ in equilibrium strictly prefers the efficient action $\hat{x}_{n}$ to all other feasible actions $x_{n} \neq \hat{x}_{n}$. It is important to note

\footnotetext{
${ }^{18}$ The notion of weak implementation is well-established in the mechanism-design literature (Palfrey 2002).
} 
that for this result to hold no special regularity assumptions on either the principals' or the agents' payoff functions are needed. The requirement that equilibrium transfers are nonzero expresses the fact that the problem is of some importance to the agents and in exchange for the efficient action something of consideration is exchanged. The latter is by the "Peppercorn Theory" (Bagwell 1995, p. 242) a prerequisite for a contract to be court enforceable.

\subsection{Action-Contingent Coordinating Contracts}

A potential drawback of the general solution to the contract-design problem of noncooperatively implementing an efficient outcome is that the equilibrium transfer schedules are generally outcomecontingent. However, we now show that under somewhat more stringent reasonable assumptions (cf. Section 2.4) on the participants' payoff functions (such as concavity) and the agents' action sets (such as convexity), supply-chain coordination may sometimes be achieved using action-contingent transfer schedules.

Theorem 4 (Action-Contingent Solution to the RCDP) Let $\hat{x} \in \mathcal{X}$ be an efficient outcome and let all principals' and all agents' payoff functions be differentiable. Then, provided that Assumption 2 and Assumption 3 are satisfied, the action-contingent excess-transfer matrix $\Delta=\left[\Delta_{n}^{m}\right]$ with

$$
\Delta_{n}^{m}\left(x_{n}\right)=\frac{\partial F^{m}(\hat{x})}{\partial x_{n}} \cdot\left(x_{n}-\hat{x}_{n}\right)-\lambda_{n}^{m}\left(x_{n}\right) \sum_{j \neq n} G_{j}\left(x_{n}, \hat{x}_{-n}\right)
$$

solves the reduced contract-design problem $(R)$ on $\mathcal{X}$ for all $(m, n) \in \mathcal{M} \times \mathcal{N}$, as long as the functions $\lambda_{n}^{m} \in C\left(\mathcal{X}_{n}, \mathbb{R}\right)$, given any outcome $x \in \mathcal{X}$, are such that

$$
\sum_{m \in \mathcal{M}} \lambda_{n}^{m}\left(x_{n}\right)=1
$$

for all $n \in \mathcal{N}$, and

$$
-\sum_{n \in \mathcal{N}} \lambda_{n}^{m}\left(x_{n}\right)\left(\sum_{j \neq n} G_{j}\left(x_{n}, \hat{x}_{-n}\right)\right) \geq F^{m}(x)-\left(\sum_{j \in \mathcal{N}} \frac{\partial F^{m}(\hat{x})}{\partial x_{j}} \cdot\left(x_{j}-\hat{x}_{j}\right)\right)
$$

for all $m \in \mathcal{M}$.

The differentiability assumption in Theorem 4 can be relaxed, since by the Rademacher theorem (Magaril-Il'yaev and Tikhomirov 2003, p. 160) payoff concavity (i.e., Assumption 2) already implies differentiability of the principals' and agents' payoffs almost everywhere. Thus, to guarantee that (25) is well defined, differentiability of the principals' payoffs is needed only at the efficient outcome $\hat{x}$ (along a path to the boundary of $\mathcal{X}$ if $\hat{x} \in \partial \mathcal{X}$, so that $\mathcal{X}$ needs to be locally pathconnected in that case). ${ }^{19}$ The solution (25) to the reduced contract-design problem in conjunction with Theorem 3 allows implementing any efficient outcome of the general multi-principal multiagent game $\Gamma$ as a WTE. Which particular WTE (in terms of the equilibrium transfer schedules)

\footnotetext{
${ }^{19}$ Even if a principal's payoff is not differentiable at the efficient outcome, it is always possible to select an appropriate element of the corresponding subdifferential.
} 
is chosen, influences the distribution of surplus in the supply chain. There is thus some interest in finding all possible solutions to the reduced contract-design problem.

Theorem 5 (Existence of an Action-Contingent Solution to the RCDP) Under the assumptions of Theorem 4 a solution of the form (25)-(27) to the reduced contract-design problem (R) exists if and only if

$$
\max _{x \in \mathcal{X}}\left\{\sum_{m \in \mathcal{M}}\left(F^{m}(x)-\sum_{j \in \mathcal{N}} \frac{\partial F^{m}(\hat{x})}{\partial x_{j}} \cdot\left(x_{j}-\hat{x}_{j}\right)\right)+\sum_{n \in \mathcal{N}} \sum_{j \neq n} G_{j}\left(x_{n}, \hat{x}_{-n}\right)\right\} \leq 0 .
$$

If we identify $F^{m}(x)-\sum_{j \in \mathcal{N}} \frac{\partial F^{m}(\hat{x})}{\partial x_{j}} \cdot\left(x_{j}-\hat{x}_{j}\right)$ as principal $m$ 's 'net affine excess payoff' and the term $\sum_{j \neq n} G_{j}\left(x_{n}, \hat{x}_{-n}\right)$ as the 'excess compliance cost' of all agents other than agent $n$ if agent $n$ implements an action $x_{n}$ possibly different from $\hat{x}_{n}$, then condition (28) can be interpreted as follows: the sum of the principals' net affine excess payoff and the agents' excess compliance cost can never exceed zero. In other words, to remain implementable a solution to the reduced contractdesign problem should be such that there is no outcome at which the principals' net affine excess payoff strictly exceeds the agents' compliance payoff (taken as negative compliance cost). Since the former is always nonpositive (cf. relation (38) in the proof of Theorem 4), this condition may be restrictive only for outcomes in which the agents' aggregate compliance cost is positive, i.e., at which there might be a positive incentive to jointly deviate. Thus, nonpositivity of the agents' excess compliance cost relative to all outcomes is sufficient for condition (28) to be satisfied. Note that in the absence of agent payoff externalities this condition holds trivially.

Corollary 5 Under the assumptions of Theorem 4 an action-contingent solution to the reduced contract-design problem $(R)$ exists if

$$
\max _{x \in \mathcal{X}}\left\{\sum_{n \in \mathcal{N}} \bar{u}_{-n}\left(x_{n}, \hat{x}_{-n}\right)\right\} \geq \sum_{n \in \mathcal{N}} u_{n}(\hat{x})
$$

where $\bar{u}_{-n}\left(x_{n}, \hat{x}_{-n}\right)=\frac{1}{N-1} \sum_{j \neq n} u_{j}\left(x_{n}, \hat{x}_{-n}\right)$ is the average gross 'compliance payoff' of agents other than agent $n$ if agent $n$ were to implement the action $x_{n} \in \mathcal{X}_{n}$ while all other agents implement the efficient action profile $\hat{x}_{-n}$.

Proof. By virtue of relation (38) in the proof of Theorem 4 condition (29) implies condition (28) in Theorem 5.

Given an action-contingent solution to the RCDP, Theorem 3 can be used as before to find a solution to the original contract-design problem; as long as the parameter functions $\vartheta_{n}^{m}$ and $\theta_{n}$ are action-contingent, the equilibrium transfer matrix $\hat{t}$ is also action-contingent.

\section{Supply-Chain Coordination}

The general results obtained in Sections 2 and 3 can be used to construct coordinating contracts in two-tier multi-principal multi-agent supply chains (cf. Figure 1). From our earlier discussions it 
is clear that the coordinating vertical contracts are such that the principals' transfers to the agents depend directly on the agents' multidimensional actions. For instance, in top-down contracting (cf. Section 2.5) a supplier's transfer to a retailer generally depends on the product quantity ordered (quantity-dependent pricing), possibly applying discounts across products and orders ("generalized tying"), and may contain clauses on the retailer's pricing policy (resale price maintenance) as well as provisions pertaining to actions for other suppliers (such as exclusive dealing). To illustrate and discuss the method, we first consider a concrete application by constructing coordinating contracts in a standard supply-chain setting (Section 4.1). We then examine a number of standard contracts often used in practical bilateral supply-chain contracting and relate them to our findings in the more general multi-principal multi-agent setting (Section 4.2).

\subsection{Application: Coordinating a Differentiated Cournot Oligopoly}

We now illustrate how to obtain closed-form expressions of coordinating contracts in a setting where $M \geq 2$ manufacturers (principals) supply differentiated goods to $N \geq 2$ retailers, who are competing as Cournot oligopolists in a common market for end-products. This is an archetypical supply-chain contracting problem of which many practical instances can be observed (such as Coke and Pepsi supplying their products to a number of retail chains), and for which no general solution is currently available in the supply-chain literature. For simplicity, we assume that each manufacturer $m$ produces its goods at a constant marginal cost $c \geq 0$. Each retailer $n$ sells the quantities $x_{n}^{m}$ it orders from manufacturer $m$ on a common market at the price

$$
p^{m}(x)=\mu-\sum_{j \in \mathcal{N}}\left(x_{j}^{m}+\beta \sum_{i \neq m} x_{j}^{i}\right) .
$$

The constant $\beta \in(-1 /(M-1), 1)$ indicates the degree to which the products are substitutes $(\beta \geq 0)$ or complements $(\beta \leq 0)$, and the constant $\mu>c$ defines the market potential. Since this is a top-down contracting problem, we can use our insights developed in Section 2.5 to transform this problem into a form that guarantees that payments (discounts) from principals to agents are nonnegative by introducing wholesale prices. Because of the symmetry in our example we can, without any loss of generality, assume that the base wholesale price $w \geq c$ is the same for all manufacturers. Manufacturer m's gross payoff (before discounts) is thus

$$
v^{m}(x ; w)=(w-c) \sum_{j \in \mathcal{N}} x_{j}^{m},
$$

while retailer $n$ 's gross payoff (before discounts) is

$$
u_{n}(x ; w)=\sum_{i \in \mathcal{M}}\left(p^{i}(x)-w\right) x_{n}^{i}
$$

Naturally, total surplus $W(x)=\sum_{i \in \mathcal{M}} v^{i}(x ; w)+\sum_{j \in \mathcal{N}} u_{j}(x ; w)=\sum_{i \in \mathcal{M}}\left(p^{i}(x)-c\right) \sum_{j \in \mathcal{N}} x_{j}^{i}$ is independent of $w$ and is maximized at the efficient outcome $\hat{x}$ with

$$
\hat{x}_{j}^{i}=\frac{\mu-c}{2 N(1+(M-1) \beta)},
$$


so that the optimal system payoff of the coordinated supply chain becomes

$$
\hat{W}=W(\hat{x})=\frac{M(\mu-c)^{2}}{4(1+(M-1) \beta)} .
$$

Since demand is unaffected by the number of agents, the surplus of the coordinated supply chain must be independent of $N$. The excess measures in (9) and (10) are thus

$$
F^{m}(x ; w)=(w-c)\left(\sum_{j \in \mathcal{N}} x_{j}^{m}-\frac{\mu-c}{2(1+(M-1) \beta)}\right)
$$

and

$$
G_{n}(x ; w)=\frac{M(\mu+c-2 w)(\mu-c)}{4 N(1+(M-1) \beta)}-\sum_{i \in \mathcal{M}}\left(p^{i}(x)-w\right) x_{n}^{i} .
$$

We obtain a general outcome-contingent ${ }^{20}$ solution to the RCDP from Corollary 1. For simplicity, we set $\varphi^{m}=\tau / M$ and $\gamma_{n}=(1-\tau) / N$ with $\tau \in C(\mathcal{X},[0,1])$ an arbitrary continuous function, so that

$$
\left\{\begin{aligned}
R^{m} & =F^{m}+\tau(\hat{W}-W) / M \\
C_{n} & =G_{n}-(1-\tau)(\hat{W}-W) / N
\end{aligned}\right.
$$

with $(m, n) \in \mathcal{M} \times \mathcal{N}$, constitutes a solution to ( $\left.\mathrm{R}^{\prime}\right)$. Using Corollary 2 it is now possible to choose from a number of different contract designs to coordinate the multi-principal multi-agent supply chain, since the choice of the excess-transfer matrix is free except for one row and column which have to be chosen in accordance with our solution to $\left(\mathrm{R}^{\prime}\right)$. Let $(i, j) \in\{1, \ldots, M-1\} \times\{1, \ldots, N-1\}$. Then, setting $\Delta_{j}^{i}=R^{i} / N$ corresponds to sharing the principals' excess payoffs (on a per-agent basis), setting $\Delta_{j}^{i}=C_{j} / M$ corresponds to sharing the agents' excess costs (on a per-principal basis), and setting $\Delta_{j}^{i}=\lambda R^{i} / N+(1-\lambda) C_{j} / M$ with $\lambda \in(0,1)$ corresponds to a convex combination of sharing the principals' excess payoffs and the agents' excess costs (on a per-supply-chain-member basis). ${ }^{21}$ Clearly more contracting possibilities do exist, but for sake of discussion we opt in this example for the third contracting option (by Lemma 3 it is also a solution), and find

$\Delta_{n}^{m}(x ; w)=\lambda(w-c)\left(\bar{x}^{i}-\hat{x}_{j}^{m}\right)+(1-\lambda)\left(\frac{\mu+c-2 w}{2} \hat{x}_{j}^{i}-\sum_{i \in \mathcal{M}} \frac{p^{i}(x)-w}{M}\left(x_{j}^{m}-\hat{x}_{j}^{m}\right)\right)+(\lambda+\tau-1) \frac{\hat{W}-W}{M N}$,

for all $(m, n) \in\{1, \ldots, M-1\} \times\{1, \ldots, N-1\}$, where $\bar{x}^{i}=(1 / N) \sum_{j \in \mathcal{N}} x_{j}^{i}$ is the average quantity ordered from principal $i$. The above excess transfers $\Delta_{n}^{m}$ are nonzero, whenever the transaction quantity between manufacturer $m$ and retailer $n$ deviates from the supply-chain average. The missing excess transfers $\Delta_{n}^{M}$ and $\Delta_{N}^{m}$ for $(m, n) \in \mathcal{M} \times \mathcal{N}$ can be obtained from (17). In general, those excess transfers will not be of exactly the same surplus-sharing form as the other excess transfers; to coordinate the supply chain at least one principal and one agent are needed as a "buffer" to balance out incentives across the supply chain. While our example exhibits a symmetric payoff structure according to (18) (including symmetric independent action sets), condition (20) is not satisfied, so that it is in fact impossible to find a completely symmetric contracting structure that coordinates our multi-principal multi-agent supply chain (cf. our discussion following Corollary 3).

\footnotetext{
${ }^{20}$ Although assumptions 2 and 3 are satisfied, condition (28) is not, so that by Theorem 5 an implementation of the efficient outcome with action-contingent contracts as in Theorem 4 is not possible.

${ }^{21}$ In our specific example, because of top-down contracting, the agents' excess cost corresponds to minus the agents' excess revenue, which can be interpreted as the agents' opportunity cost when implementing inefficient actions.
} 
We are now ready to use Theorem 3 and specify a coordinating contracting structure. To simplify expressions we set $\vartheta_{n}^{m} \equiv 1 / M$, and $\theta_{n} \equiv \theta \in[0,1]$ small enough, so that for $(m, n) \in\{1, \ldots, M-$ $1\} \times\{1, \ldots, N-1\}:$

$\hat{\Delta}_{n}^{m}(x ; w)=\lambda(w-c)\left(\bar{x}^{i}-\hat{x}_{j}^{m}\right)+(1-\lambda)\left(\frac{\mu+c-2 w}{2} \hat{x}_{j}^{i}-\sum_{i \in \mathcal{M}} \frac{p^{i}(x)-w}{M}\left(x_{j}^{m}-\hat{x}_{j}^{m}\right)\right)+(\lambda+\tau-1+\xi) \frac{\hat{W}-W}{M N}$,

where $\xi=(1-\tau)(1-\theta)$. The remaining elements of $\hat{\Delta}$ are $\hat{\Delta}_{n}^{M}=\Delta_{n}^{M}+\xi(\hat{W}-W) /(M N)$ and $\hat{\Delta}_{N}^{m}=\Delta_{N}^{m}+\xi(\hat{W}-W) /(M N)$ for all $(m, n) \in \mathcal{M} \times \mathcal{N}$. Thus, using (21) a set of coordinating contracts can be specified in the form

$$
\hat{t}_{n}^{m}(x ; w)=\hat{\Delta}_{n}^{m}(x ; w)-\min _{x_{n} \in \mathcal{X}_{n}}\left\{\hat{\Delta}_{n}^{m}\left(x_{n}, \hat{x}_{-n} ; w\right)\right\},
$$

where retailer $n$ 's action set $\mathcal{X}_{n}$ can, without any loss in generality, be restricted to all nonnegative order quantities that do not exceed the maximally plausible 'monopolistic' order quantity $(\mu-$ $c) /(2(1+(M-1) \beta))$ when all other retailers order nothing. ${ }^{22}$ We obtain the in-equilibrium discounts,

$$
\alpha_{n}^{m}(w)=-\min _{x_{n} \in \mathcal{X}_{n}}\left\{\hat{\Delta}_{n}^{m}\left(x_{n}, \hat{x}_{-n} ; w\right)\right\} \in\left[0, w \hat{x}_{n}^{m}\right],
$$

offered by manufacturer $m$ to agent $n$, where we have set $\theta=0$ for simplicity and assumed that $w$ is chosen large enough, $\hat{x}_{n}^{m} w \geq \alpha_{n}^{m}(w)$, to provide the appropriate incentives through the nonnegative discounts. By Corollary 4 one obtains a strong implementation for any arbitrarily small positive $\theta$, so that it is possible to strongly implement the unique efficient outcome using in-equilibrium discounts arbitrarily close to the above.

It is important to note that it is impossible to coordinate the supply chain using wholesale prices only (even when they vary with $m$ and $n$ ). The nonlinearities in the above equilibrium discount schedules are essential for the noncooperative provision of appropriate incentives for the retailers to implement the efficient outcome.

\subsection{Vertical Contracting Provisions}

In his excellent review of vertical contracting, Katz (1989) outlines six functions of vertical contracts including quantity-dependent pricing, ties, royalty schemes, requirements contracts, resale price restraints, and resale customer restraints. We discuss how our framework can accommodate each of these functions and examine practical complete-information contracts ("commercial contracts") that are often used in supply-chain settings (Cachon 2003).

Quantity-Dependent Pricing. Nonlinear transfer schedules can serve both to price discriminate in situations with asymmetric information (screening contracts) and to coordinate a supply chain. In our complete-information setting we naturally limit our attention to coordination. Oren et al. (1982) demonstrated the powerful role of two-part tariffs in quantity-dependent pricing. In fact, as a direct consequence of convex analysis, as long as a nonlinear pricing schedule is concave it can be represented as the lower envelope of an indexed family of two-part tariffs. Jeuland and

\footnotetext{
${ }^{22}$ In a Cournot oligopoly the players' actions are strategic substitutes.
} 
Shugan (1983) show that nonlinear pricing schemes can coordinate a channel, and Moorthy (1987) points out that indeed this can be achieved using simple two-part tariffs. Our findings confirm that Moorthy's intuition carries over to multi-principal multi-agent environments as long as there are no agent-payoff externalities and all payoff functions are concave. In that case, we obtain by Theorem 4 and Theorem 5 action-contingent, affine contracts, corresponding to two-part tariffs. However, when there are agent-payoff externalities the efficiency of two-part tariffs generally breaks down, as our example in Section 4.1 shows. Similarly, when payoff functions are nonconcave, the separating hyperplane theorem ceases to hold and it may become impossible to coordinate the supply chain using two-part tariffs. In the general case a coordinating quantity-dependent pricing contract becomes nonlinear.

Ties. Given that each agent's action can be $L$-dimensional, it is possible for $L>1$ that principal $m$ 's equilibrium transfers are not additively separable in the different components, $x_{n, 1}^{m}, \ldots, x_{n, L}^{m}$, of agent $n$ 's action. In that case, the compensation for different components of an agent's action is linked, which amounts to a (generalized) tying arrangement. Tying arrangements in this sense may arise naturally as a consequence of (anti-)complementarities in an agent's cost structure, as can easily be seen from the coordinating contracts in Theorem 3: tying can occur whenever $G_{n}$ or $F^{m}$ lack additive separability, resulting in interdependencies of incentives at the margin. We emphasize that tying arrangements under these circumstances are efficient in the sense of maximizing total surplus of the supply chain.

Royalty Schemes. In contracts with royalty schemes, the transfer payment between a supplier and a buyer is a function of the buyer's sales in the final goods market rather than based on the amount of intermediary goods exchanged. In that situation, some of the contractual provisions may be contingent on the resolution of a random variable (such as demand). Although ex-post provisions can be accommodated in our framework, we emphasize that at least in the absence of renegotiation all the contractual terms are constructed based on all parties' expected payoffs. Our method yields certainty-equivalent contracts which are ex-ante coordinating and may without loss of generality contain ex-post provisions. ${ }^{23}$ However, ex-post provisions that result in the same expected payoffs (or expected utilities in the presence of risk aversion) are all equivalent, and thus we cannot expect specific ex-post design statements from our theory, but we can nonetheless integrate existing contractual schemes that contain ex-post provisions (such as quantity-flexibility contracts) into a unifying framework. The following commercial contracts with royalty schemes are commonly found in supply chains. These contracts tend to be used in markets for goods with a relatively short shelf life, such as periodicals, baked goods, video rentals, or current car models.

- Pay-Back Contract. One possibility for a supplier to coordinate a one-to-many supply chain recognized by Pasternack (1985) is the pay-back contract, in which retailer $m$ pays supplier $n$ an amount of $w_{n}^{m}$ per unit purchased, plus $b_{n}^{m}$ per unit remaining at the end of the season in order to incentivize the supplier's capacity investment. This contract is essentially a two-part tariff scheme and thus our earlier remarks on quantity-dependent pricing apply.

\footnotetext{
${ }^{23}$ If $\tilde{\omega}$ is a random variable and $\tau_{n}^{m}\left(x_{n}, \tilde{\omega}\right)$ is an ex-post contingent contract between principal $m$ and agent $n$, then our theory makes statements about the certainty-equivalent contract $t_{n}^{m}\left(x_{n}\right)=E \tau_{n}^{m}\left(x_{n}, \tilde{\omega}\right)$.
} 
- Revenue-Sharing Contract. Cachon and Lariviere (2005) discuss revenue sharing as a way to coordinate supply chains in which reliable revenue monitoring is feasible, such as in the market for video rentals. Under such a revenue-sharing scheme retailer $m$ pays supplier $n$ an amount of $w_{n}^{m}$ per unit capacity installed, plus a fraction of his revenue. In our framework, revenuesharing, cost-sharing, or surplus sharing contracts arise naturally from the solution to the reduced contract design problem in corollaries 1 and 2 (cf. also the example in Section 4.1). From a coordination standpoint, the choice of the solution $\Delta_{-N}^{-M}$ is essentially free, but it critically influences the symmetry of transfer agreements across the supply chain. If actiondependent contracts can be implemented in the multi-principal multi-agent supply chain (using results from Section 3.3), the resulting contracts have the flavor of both sharing the principals' revenues linearly, and sharing the agents' revenues as a function of the externalities that agent $n$ exerts on the other agents.

- Quantity-Flexibility Contract. Tsay (1999) studies supply chain coordination with quantityflexibility contracts. Under a quantity-flexibility contract mechanism, retailer $m$ pays supplier $n$ an amount of $w_{n}^{m}$ per unit purchased and compensates the supplier for unused capacity up to a fraction $\rho_{n}^{m} \in[0,1]$ of total capacity installed.

- Sales-Rebate Contract. Taylor (2002) considers a sales-rebate contract for supply-chain coordination, under which retailer $m$ pays supplier $n$ an amount of $w_{n}^{m}$ per unit purchased and an extra rebate $r_{n}^{m}$ per unit sold above a threshold $q_{n}^{m}$. This corresponds to a quantity-dependent transfer scheme which can naturally arise in our framework.

Requirements Contracts. Bilateral contracts that contain provisions which affect an agent's payoff with respect to his behavior across different principals are generally termed requirements contracts. In the extreme, requirements contracts could involve exclusive dealing arrangements, in which certain agents exclusively trade with certain principals and are compensated accordingly. In cases where agents each implement multiple actions, such as capacity orders and pricing decisions, requirements contracts may also include resale price restraints (e.g., to achieve a price maintenance level) or resale customer restraints (e.g., to guarantee territoriality and thus restrict agent competition detrimental to overall supply-chain profit). As a direct consequence of Theorem 3 , to implement efficient outcomes in multi-principal multi-agent supply chains, requirements contracts are generally unavoidable (except possibly when assumptions 2 and 3 hold). These requirements contracts, even though they arise noncooperatively, might be interpreted by a regulator as anticompetitive behavior.

\section{Conclusion}

Supply chains with many participating firms are a ubiquitous reality. It may therefore seem surprising that the coordination of such supply chains has received virtually no attention in the extant literature. This lack of results is most likely not due to oversight but to the associated technical difficulties which have been partially overcome by recent advances in the economics literature. Il- 
lustrating the additional complications, Cachon's (2003) postulate that "[e]ach firm in a supply chain must execute a precise set of actions to achieve optimal supply-chain performance" carries a new meaning in multi-principal multi-agent supply chains, since maximizing the firms' overall surplus requires not only vertical coordination through contracts but also implicit horizontal coordination, however without the use of anticompetitive practices. It is the noncooperative nature of the principals' contract design that introduces a number of technical difficulties which may account for the current lack of results in multi-principal multi-agent supply-chain contracting, a gap which we hope to fill: as long as all payoffs are continuous (in a weak sense, allowing for discrete action sets; cf. footnote 10) on compact action sets, we have provided a set of contracts that coordinate any multi-principal multi-agent supply chain. More specifically, starting from a solution to a "reduced contract-design problem" (Theorem 2), we have shown that it is possible to obtain coordinating contracts which allocate surplus to the different participants in the supply chain by assigning appropriate outcome-contingent weights in the simple transformation (Theorem 3) leading to coordinating bilateral contracts in terms of (nonlinear) transfer-payment schedules. We emphasize that in contrast to most of the available results, even in the literature on single-principal single-agent supply-chain contracting, our approach is entirely nonparametric: weighting functions are used solely to select particular elements from the set of coordinating contracts. In addition to filling a void in the theoretical literature on supply-chain contracting, our results have a number of interesting practical implications, of which we stress only two.

- First, virtually all known (and used) commercial contracts can be employed by (almost all) principals to coordinate multi-principal multi-agent supply chains, since Corollary 2 provides large flexibility in conjunction with Corollary 1 (e.g., by setting the $\gamma_{n}$ 's to zero). To achieve reasonably homogeneous contracting terms across the supply chain, however, the contracts in general will contain a portion of surplus which translates nonlinearities in payoffs to nonlinearities in incentive payments. The results on single-principal single-agent supply chains are thus naturally nested in our more general framework. However, our results imply that in general, to coordinate a multi-principal multi-agent supply chain one principal and one agent need to be used as a "buffer" to distribute incentives across all members of the supply chain (cf. Corollary 2). If all payoffs are concave and all action sets are convex, it may be possible to coordinate the supply chain using action-contingent contracts. The corresponding results in Section 3.3 generalize earlier results by P\&R and Strulovici and Weber (2004).

- Second, affine contracts - even though easy to write down - are generally not desirable to principals: for any given agent $n$, the more a principal is able to promise out of equilibrium, the less she has to pay this agent in equilibrium! In other words, in-equilibrium transfers are dramatically related to the out-of-equilibrium contract design. However, since in order to enable supply-chain coordination the total amount of out-of-equilibrium promises is limited for each agent (to $G_{n}$ ), each principal has a vested interest to capture as much of these feasible promises as possible. As a result, the allocation of the (outcome-contingent) weights $\vartheta_{n}^{m}$ (which sum to one over all $m \in \mathcal{M}$ ) is most likely subject to negotiation, despite the fact that the actual equilibrium implementation is by construction noncooperative and is thus not in 
conflict with anticompetitive clauses.

The results obtained here are limited in the sense that it is critical that contracts can be made contingent on all agents' actions. It is thus important that these actions be observable by all members of the supply chain and verifiable by an independent court of law, which guarantees timely enforcement. Further research is needed to provide satisfying answers in more realistic settings. Another limitation, and thus an opportunity for further research, lies in our nonexhaustive answer to the question of selecting "appropriate" coordinating contracts out of the set of feasible coordinating contracts. Depending on the situation, this selection might be guided by aspects such as revenue extraction or practical implementability. For instance, under customary compliance regimes in a certain industry some contracts might be preferred to others; or, some contracts (e.g., the ones without requirements clauses) may allow for simpler monitoring given the industry specifics. The last points us to another major research direction related to multi-principal multi-agent contracting in which we foresee much activity: the relaxation of the full information (or full contractibility) assumption, allowing for moral hazard and/or hidden information in supply-chain relationships, be they vertical or horizontal.

\section{References}

[1] Aubin, J.-P., Ekeland, I. (1984) Applied Nonlinear Analysis, Wiley, New York, NY.

[2] Bagwell, C.E. (1995) Managers and the Legal Environment, West Publishing, San Francisco, CA.

[3] Berge, C. (1963) Topological Spaces, Oliver and Boyd, Edinburgh, UK. Reprinted by Dover Publications, Mineola, NY, in 1997.

[4] Bernheim, B.D., Whinston, M.D. (1986) "Menu Auctions, Resource Allocations, and Economic Influence," Quarterly Journal of Economics, Vol. 101, No. 1, pp. 1-31.

[5] Bernheim, B.D., Peleg, B., Whinston, M.D. (1987) "Coalition Proof Nash Equilibria, I: Concepts," Journal of Economic Theory, Vol. 42, No. 1, pp. 1-12.

[6] Bernstein, F., Federgruen, A. (2005) "Decentralized Supply Chains with Competing Retailers Under Demand Uncertainty," Management Science, Vol. 51, No. 1, pp. 18-29.

[7] Bertsekas, D.P. (1995) Nonlinear Programming, Athena Scientific, Belmont, MA.

[8] Cachon, G. (2003) "Supply Chain Coordination with Contracts," in: Graves, S., de Kok, T. (eds.), Handbooks in Operations Research and Management Science: Supply Chain Management, North-Holland, Amsterdam, NL, pp. 229-339.

[9] Cachon, G., Lariviere, M. (2005) "Supply Chain Coordination with Revenue-Sharing Contracts: Strengths and Limitations," Management Science, Vol. 51, No. 1, pp. 30-44.

[10] Carr, S.M., Karmarkar, U.S. (2005) "Competition in Multi-Echelon Assembly Supply Chains", Management Science, Vol. 51, No. 1, pp. 45-59.

[11] Grossman, S.J., Hart, O.D. (1986) "The Costs and Benefits of Ownership: A Theory of Vertical and Lateral Integration," Journal of Political Economy, Vol. 94, No. 4, pp. 691-719.

[12] Jensen, M.C., Meckling, W.H. (1976) "Theory of the Firm: Managerial Behavior, Agency Costs and Ownership Structure," Journal of Financial Economics, Vol. 3, No. 4, pp. 305-360. 
[13] Jeuland, A.P., Shugan, S.M. (1983) "Managing Channel Profits," Marketing Science, Vol. 2, No. 3, pp. 239-272.

[14] Katz, M.L. (1989) "Vertical Contractual Relations," in: Schmalensee, R., Willig, R.D. (eds.), Handbook of Industrial Organization, Vol. 1, North Holland, Amsterdam, NL, pp. 655-721.

[15] Magaril-Il'yaev, G.G., Tikhomirov, V.M. (2003) Convex Analysis: Theory and Applications, American Mathematical Society, Providence, RI.

[16] Majumder, P., Srinivasan, A. (2003) "Asset-Specific Supply Tree Competition and Leadership," Working Paper, Fuqua School of Business, Duke University, Durham, NC.

[17] Maskin, E., Tirole, J. (1990) "The Principal-Agent Relationship with an Informed Principal: The Case of Private Values," Econometrica, Vol. 58, No. 2, pp. 379-409.

[18] Moorthy, K.S. (1987) "Managing Channel Profits: Comment," Marketing Science, Vol. 6, No. 4, pp. $375-379$.

[19] Oren, S.S., Smith, S.A., Wilson, R.B. (1982) "Nonlinear Pricing in Markets with Interdependent Demand," Marketing Science, Vol. 1, No. 3, pp. 287-313.

[20] Palfrey, T.R. (2002) "Implementation Theory," in: Auman, R.J., Hart, S., Handbook of Game Theory, Vol. 3, Elsevier Science, Amsterdam, NL, pp. 2271-2326.

[21] Pasternack, B.A. (1985) "Optimal Pricing and Return Policies For Perishable Commodities," Marketing Science, Vol. 4, No. 2, pp. 166-176.

[22] Prat, A., Rustichini, A. (2003) "Games Played Through Agents," Econometrica, Vol. 71, No. 4, pp. 989-1026.

[23] Segal, I. (1999) "Contracting With Externalities," Quarterly Journal of Economics, Vol. 104, No. 2, pp. 337-388.

[24] Segal, I., Whinston, M.D. (2003) "Robust Predictions For Bilateral Contracting with Externalities," Econometrica, Vol. 71, No. 3, pp. 757-791.

[25] Spengler, J.J. (1950) "Vertical Integration and Antitrust Policy," Journal of Political Economy, Vol. 58, No. 4, pp. 347-352.

[26] Strulovici, B.H., Weber, T.A. (2004) "Constructing Efficient Equilibria in Games Played Through Agents," Working Paper, Department of Management Science and Engineering, Stanford University, Stanford, CA.

[27] TAYlor, T. (2002) "Coordination Under Channel Rebates with Sales Effort Effects," Management Science, Vol. 48, No. 8, pp. 992-1007.

[28] Tsay, A.A. (1999) "The Quantity Flexibility Contract and Supplier-Customer Incentives," Management Science, Vol. 45, No. 10, pp. 1339-1358.

[29] Von Neumann, J., Morgenstern, O. (1944) Theory of Games and Economic Behavior, Princeton University Press, Princeton, NJ.

\section{Appendix: Proofs}

Proof of Lemma 1. Since $\hat{t}^{m}$ is weakly truthful with respect to the equilibrium outcome $\hat{x}$, we obtain by Definition 3 that

$$
v^{m}(\hat{x})-\sum_{n \in \mathcal{N}} \hat{t}_{n}^{m}(\hat{x}) \geq v^{m}(x)-\sum_{n \in \mathcal{N}} \hat{t}_{n}^{m}(x)
$$

for all $x \in \mathcal{X}$ and all $m \in \mathcal{M}$. By virtue of the inclusion (4), which characterizes the second-stage purestrategy Nash equilibrium, we have that

$$
u_{n}(\hat{x})+\sum_{m \in \mathcal{M}} \hat{t}_{n}^{m}(\hat{x}) \geq u_{n}\left(x_{n}, \hat{x}_{-n}\right)+\sum_{m \in \mathcal{M}} \hat{t}_{n}^{m}\left(x_{n}, \hat{x}_{-n}\right)
$$


for all $x_{n} \in \mathcal{X}_{n}$ and all $n \in \mathcal{N}$. Hence, by summing up relation (30) over all $m \in \mathcal{M}$ and relation (31) over all $n \in \mathcal{N}$ we obtain that relation (7) in Definition 2 holds, so that the outcome $\hat{x}$ is efficient relative to the equilibrium transfer schedule $\hat{t}^{24}$

Proof of Theorem 1. The equilibrium condition (4) can be equivalently rewritten in the form (AM). Principal $m$, given the other principals' transfer vector $\hat{t}^{-m}$, can induce agents to implement any outcome $x \in$ $\mathcal{X}$, if only she promises each agent $n$ a transfer that is larger than the difference the agent would obtain by implementing his otherwise preferred action. In other words, to persuade agent $n$ to implement the outcome $x_{n}$ which is part of her desired overall outcome $x=\left(x_{1}, \ldots, x_{N}\right)$, principal $m$ 's transfer $\hat{t}_{n}^{m}\left(x_{n}, \hat{x}_{-n}\right)$ to agent $n$ as a reward for action $x_{n}$ needs to satisfy

$$
\begin{aligned}
\hat{t}_{n}^{m}\left(x_{n}, \hat{x}_{-n}\right) & \geq \max _{\xi_{n} \in \mathcal{X}_{n}}\left\{u_{n}\left(\xi_{n}, \hat{x}_{-n}\right)+\sum_{i \neq m} \hat{t}_{n}^{i}\left(\xi_{n}, \hat{x}_{-n}\right)\right\}-\left(u_{n}\left(x_{n}, \hat{x}_{-n}\right)+\sum_{i \neq m} \hat{t}_{n}^{i}\left(x_{n}, \hat{x}_{-n}\right)\right) \\
& =R_{n}^{m}\left(\hat{x}_{-n}\right)-\left(u_{n}\left(x_{n}, \hat{x}_{-n}\right)+\sum_{i \neq m} \hat{t}_{n}^{i}\left(x_{n}, \hat{x}_{-n}\right)\right)
\end{aligned}
$$

where

$$
R_{n}^{m}\left(\hat{x}_{-n}\right)=\max _{\xi_{n} \in \mathcal{X}_{n}}\left\{u_{n}\left(\xi_{n}, \hat{x}_{-n}\right)+\sum_{i \neq m} \hat{t}_{n}^{i}\left(\xi_{n}, \hat{x}_{-n}\right)\right\}
$$

is agent n's highest incremental (i.e., over and above what he obtains by simply free-riding on the other agents' actions) reward without principal $m$. As a consequence of the definition of weak truthfulness (Definition 3), any equilibrium outcome $\hat{x}$ needs to maximize principal $m$ 's net payoff,

$$
\begin{aligned}
V^{m}\left(x, \hat{t}^{m}\right) & =v^{m}(x)-\sum_{n \in \mathcal{N}} \hat{t}_{n}^{m}\left(x_{n}, \hat{x}_{-n}\right) \\
& \geq v^{m}(x)-\sum_{n \in \mathcal{N}}\left(R_{n}^{m}\left(\hat{x}_{-n}\right)-\left(u_{n}\left(x_{n}, \hat{x}_{-n}\right)+\sum_{i \neq m} \hat{t}_{n}^{i}\left(x_{n}, \hat{x}_{-n}\right)\right)\right),
\end{aligned}
$$

so that $\hat{x}$ solves the fixed-point problem

$$
\hat{x} \in \arg \max _{x \in \mathcal{X}}\left\{v^{m}(x)+\sum_{n \in \mathcal{N}}\left(u_{n}\left(x_{n}, \hat{x}_{-n}\right)+\sum_{i \neq m} \hat{t}_{n}^{i}\left(x_{n}, \hat{x}_{-n}\right)\right)\right\} .
$$

Let us now consider principal $m$ 's equivalent cost minimization problem. Given an outcome $\check{x}$ principal $m$ solves

$$
\min _{t^{m} \in C\left(\mathcal{X}, \mathcal{T}^{m}\right)} \sum_{n \in \mathcal{N}} t_{n}^{m}\left(\check{x}_{n}, \hat{x}_{-n}\right),
$$

subject to

$$
t_{n}\left(\check{x}_{n}, \hat{x}_{-n}\right)+u_{n}\left(\check{x}_{n}, \hat{x}_{-n}\right)+\sum_{i \neq m} t_{n}^{i}\left(\check{x}_{n}, \hat{x}_{-n}\right) \geq t_{n}\left(x_{n}, \hat{x}_{-n}\right)+u_{n}\left(x_{n}, \hat{x}_{-n}\right)+\sum_{i \neq m} t_{n}^{i}\left(x_{n}, \hat{x}_{-n}\right),
$$

for all $x_{n} \in \mathcal{X}_{n}$ and all $n \in \mathcal{N}$. One can verify that any solution $\hat{t}^{m}$ to the cost minimization problem (33)-(34) is such that

$$
\hat{t}_{n}^{m}\left(\check{x}_{n}, \hat{x}_{-n}\right)=R_{n}^{m}\left(\hat{x}_{-n}\right)-\left(u_{n}\left(\check{x}_{n}, \hat{x}_{-n}\right)+\sum_{i \neq m} \hat{t}_{n}^{i}\left(\check{x}_{n}, \hat{x}_{-n}\right)\right)
$$

and

$$
\hat{t}_{n}^{m}\left(\check{x}_{n}, \hat{x}_{-n}\right) \leq R_{n}^{m}\left(\hat{x}_{-n}\right)-\left(u_{n}\left(\check{x}_{n}, \hat{x}_{-n}\right)+\sum_{i \neq m} \hat{t}_{n}^{i}\left(x_{n}, \hat{x}_{-n}\right)\right)
$$

\footnotetext{
${ }^{24}$ This also implies the more restrictive inequality in footnote 9.
} 
for all $x_{n} \in \mathcal{X}_{n}$. By replacing $\left(\check{x}_{n}, \hat{x}_{-n}\right)$ with the equilibrium outcome $\hat{x}$ we obtain

$$
u_{n}(\hat{x})+\sum_{i \in \mathcal{M}} \hat{t}_{n}^{i}(\hat{x})=R_{n}^{m}\left(\hat{x}_{-n}\right)
$$

and

$$
u_{n}\left(x_{n}, \hat{x}_{-n}\right)+\sum_{i \in \mathcal{M}} \hat{t}_{n}^{i}\left(x_{n}, \hat{x}_{-n}\right) \leq R_{n}^{m}\left(\hat{x}_{-n}\right)
$$

for all $x_{n} \in \mathcal{X}_{n}$. The last two relations together are equivalent to (PM).

$\Leftarrow$ : Let $(\hat{t}, \hat{x}) \in C(\mathcal{X}, \mathcal{T}) \times \mathcal{X}$ be a pair that satisfies (WT), (AM), (PM). The inequality (WT) implies by Definition 3 that $\hat{t}^{m}$ is weakly truthful for any principal $m \in \mathcal{M}$. It is clear that (AM) is equivalent to (4), i.e., given $\hat{t}$ the action $\hat{x}_{n}$ is a best response for any agent $n \in \mathcal{N}$. By summing up (AM) over all $n \in \mathcal{N}$ and adding (WT) we obtain

$$
v^{m}(\hat{x})+\sum_{n \in \mathcal{N}}\left(u_{n}(\hat{x})+\sum_{i \neq m} \hat{t}_{n}^{i}(\hat{x})\right) \geq v^{m}(x)+\sum_{n \in \mathcal{N}}\left(u_{n}\left(x_{n}, \hat{x}_{-n}\right)+\sum_{i \neq m} \hat{t}_{n}^{i}\left(x_{n}, \hat{x}_{-n}\right)\right)
$$

for all principals $m \in \mathcal{M}$ and outcomes $x \in \mathcal{X}$. The latter inequality is equivalent to (32), which in turn implies (5). Thus, the pair $(\hat{t}, \hat{x})$ must be a WTE of the game $\Gamma$.

Proof of Lemma 2. (i) At the efficient outcome $\hat{x}$ we have that $F^{m}(\hat{x})=G_{n}(\hat{x})=0$ for all $(m, n) \in \mathcal{M} \times \mathcal{N}$. Hence, the matrix $\Delta(\hat{x})=\left[\Delta_{j}^{i}(\hat{x})\right]$ must be such that

$$
\sum_{i \in \mathcal{M}} \Delta_{n}^{i}(\hat{x}) \leq 0 \leq \sum_{j \in \mathcal{N}} \Delta_{j}^{m}(\hat{x})
$$

for all $(m, n) \in \mathcal{M} \times \mathcal{N}$. Assume that there exists an index $\hat{m} \in \mathcal{M}$ such that $\sum_{j \in \mathcal{N}} \Delta_{j}^{\hat{m}}(\hat{x})>0$. By separately summing up all the rows and summing up all the columns of $\Delta(\hat{x})$ we obtain

$$
\sum_{n \in \mathcal{N}} \sum_{i \in \mathcal{M}} \Delta_{n}^{i}(\hat{x}) \leq 0<\sum_{m \in \mathcal{M}} \sum_{j \in \mathcal{N}} \Delta_{j}^{m}(\hat{x})
$$

a contradiction. As a result $\sum_{j \in \mathcal{N}} \Delta_{j}^{\hat{m}}(\hat{x})=0$ for all $m \in \mathcal{M}$. We can show in an analogous manner that necessarily $\sum_{i \in \mathcal{M}} \Delta_{n}^{i}(\hat{x})=0$, whence relation (12) obtains. (ii) $\Rightarrow$ : The continuous matrix function $\Delta$ satisfies (R). Since in addition (12) holds at $\hat{x}$ and $\delta$ is a constant matrix by assumption, it must be true that

$$
\sum_{i \in \mathcal{M}} \delta_{n}^{i} \leq 0 \leq \sum_{j \in \mathcal{N}} \delta_{j}^{m}
$$

for all $(m, n) \in \mathcal{M} \times \mathcal{N}$. Thus, as in part (i) we can conclude that relation (13) necessarily holds. $\Leftarrow$ : Substituting (13) into the reduced contract design inequalities for $\Delta+\delta$ yields the same inequalities as if we had set $\delta=0$. Since $\Delta$ solves the reduced contract-design problem (R) by assumption, the matrix function $\Delta+\delta$ constitutes also a solution to the reduced contract-design problem.

Proof of Lemma 3. Fix a $\lambda \in(0,1)$. Then any two solutions $\Delta=\left[\Delta_{n}^{m}\right]$ and $\tilde{\Delta}=\left[\tilde{\Delta}_{n}^{m}\right]$ to the reduced contract-design problem $(\mathrm{R})$ satisfy

$$
\lambda\left(F^{m}-\sum_{j \in \mathcal{N}} \Delta_{j}^{m}\right)+(1-\lambda)\left(F^{m}-\sum_{j \in \mathcal{N}} \tilde{\Delta}_{j}^{m}\right) \leq 0 \leq \lambda\left(G_{n}-\sum_{i \in \mathcal{M}} \Delta_{n}^{i}\right)+(1-\lambda)\left(G_{n}-\sum_{i \in \mathcal{M}} \tilde{\Delta}_{n}^{i}\right),
$$

for all $(m, n) \in \mathcal{M} \times \mathcal{N}$, which concludes our proof. 
Proof of Theorem 2. Since the outcome $\hat{x} \in \mathcal{X}$ is by assumption efficient, the excess welfare $W(x)-W(\hat{x})$ relative to any outcome $x \in \mathcal{X}$ is nonpositive. On the other hand, problem (R') can be equivalently rewritten in the form

$$
\left\{\begin{array}{c}
f^{m}(x) \equiv R^{m}(x)-F^{m}(x) \geq 0 \\
g_{n}(x) \equiv G_{n}(x)-C_{n}(x) \geq 0, \\
\sum_{i \in \mathcal{M}} f^{i}(x)+\sum_{j \in \mathcal{N}} g_{j}(x)=W(\hat{x})-W(x),
\end{array}\right.
$$

for all $(m, n) \in \mathcal{M} \times \mathcal{N}$. Since the solution $\left(R^{1}, \ldots, R^{M}\right),\left(C_{1}, \ldots, C_{N}\right)$ to (R') is by assumption continuous, the functions $f^{m}, g_{n}$ defined in (35) are elements of $C\left(\mathcal{X}, \mathbb{R}_{+}\right)$, which concludes our proof.

Proof of Theorem 3. Let $\hat{x} \in \arg \max _{x \in \mathcal{X}} W(x)$ be a given efficient outcome. By Theorem 1 the pair $(\hat{t}, \hat{x})$ with $\hat{t}_{n}^{m}(x)$ as defined in (21)-(22) constitutes a WTE if and only if it satisfies the conditions (WT), (AM), and (PM). Consider first (WT), which is equivalent to (WT'). Since $\Delta$ by assumption satisfies (WT'), we have by (22) that $\hat{\Delta}_{n}^{m} \geq \Delta_{n}^{m}$, so that

$$
F^{m}(x)-\sum_{j \in \mathcal{N}} \hat{\Delta}_{j}^{m}\left(x ; \vartheta_{n}^{m}, \theta_{n}\right) \leq F^{m}(x)-\sum_{j \in \mathcal{N}} \Delta_{j}^{m}(x) \leq 0
$$

for all $m \in \mathcal{M}$ and all $x \in \mathcal{X}$. Thus, $\hat{\Delta}$ satisfies (WT). To show that it also satisfies (AM), which is equivalent to (AM'), we simply note that

$$
G_{n}\left(x_{n}, \hat{x}_{n}\right)-\sum_{i \in \mathcal{M}} \hat{\Delta}_{n}^{i}\left(x_{n}, \hat{x}_{n} ; \vartheta_{n}^{i}, \theta_{n}\right)=\left(1-\sum_{i \in \mathcal{M}} \eta_{n}^{i}\left(x_{n}, \hat{x}_{-n}\right)\right)\left(G_{n}\left(x_{n}, \hat{x}_{-n}\right)-\sum_{i \in \mathcal{M}} \Delta_{n}^{i}\left(x_{n}, \hat{x}_{-n}\right)\right) \geq 0
$$

for all $n \in \mathcal{N}$ and all $x \in \mathcal{X}$ (where $\eta_{n}^{m}=\vartheta_{n}^{m}\left(1-\theta_{n}\right)$ ), since $\Delta$ satisfies (AM') by hypothesis. Lastly, we consider (PM), which can be rewritten in terms of excess measures as follows:

$$
\hat{t}_{n}^{m}(\hat{x})=\max _{x_{n} \in \mathcal{X}_{n}}\left\{-G_{n}\left(x_{n}, \hat{x}_{-n}\right)+\sum_{i \neq m} \hat{\Delta}_{n}^{i}\left(x_{n}, \hat{x}_{-n}\right)\right\} .
$$

Note that by substituting (22) we have that

$$
\begin{aligned}
-G_{n}(x)+\sum_{i \neq m} \hat{\Delta}_{n}^{i}\left(x ; \vartheta_{n}^{m}, \theta_{n}\right) & =-\Delta_{n}^{m}(x)-\left(\sum_{i \in \mathcal{M}} \vartheta_{n}^{i}(x) \theta_{n}(x)+\vartheta_{n}^{m}(x)\left(1-\theta_{n}(x)\right)\right)\left(G_{n}(x)-\sum_{i \in \mathcal{M}} \Delta_{n}^{i}(x)\right) \\
& =-\Delta_{n}^{m}(x)-\left(\vartheta_{n}^{m}(x)+\theta_{n}(x)-\vartheta_{n}^{m}(x) \theta_{n}(x)\right)\left(G_{n}(x)-\sum_{i \in \mathcal{M}} \Delta_{n}^{i}(x)\right) \\
& =-\hat{\Delta}_{n}^{m}\left(x ; \vartheta_{n}^{m}+\theta_{n}, \vartheta_{n}^{m} \theta_{n} /\left(\vartheta_{n}^{m}+\theta_{n}\right)\right)
\end{aligned}
$$

for all $(m, n) \in \mathcal{M} \times \mathcal{N}$ and all $x \in \mathcal{X} .{ }^{25}$ We therefore obtain that (PM') is equivalent to

$$
\hat{t}_{n}^{m}\left(\hat{x} ; \vartheta_{n}^{m}, \theta_{n}\right)=-\min _{x_{n} \in \mathcal{X}_{n}}\left\{\hat{\Delta}_{n}^{m}\left(x ; \vartheta_{n}^{m}+\theta_{n}, \vartheta_{n}^{m} \theta_{n} /\left(\vartheta_{n}^{m}+\theta_{n}\right)\right)\right\}
$$

an expression that always exists by the Weierstrass theorem (Bertsekas 1995, p. 540), since $\mathcal{X}_{n}$ is by assumption compact and $\hat{\Delta}_{n}^{m}$ is continuous. If we set $\alpha_{n}^{m}\left(\vartheta_{n}^{m}, \theta_{n}^{m}\right)=\hat{t}_{n}^{m}\left(\hat{x} ; \vartheta_{n}^{m}, \theta_{n}\right)$, then by (21) it is

$$
\hat{t}_{n}^{m}\left(x ; \vartheta_{n}^{m}, \theta_{n}\right)=\hat{\Delta}_{n}^{m}\left(x ; \vartheta_{n}^{m}, \theta_{n}\right)+\alpha_{n}^{m}\left(\vartheta_{n}^{m}, \theta_{n}\right)
$$

for all $(m, n) \in \mathcal{M} \times \mathcal{N}$ and all $x \in \mathcal{X}$. Furthermore, $\hat{\Delta}_{n}^{m}\left(x ; \vartheta_{n}^{m}, 0\right) \geq-\alpha_{n}^{m}\left(\vartheta_{n}^{m}, 0\right)$, so that

$$
0 \in\left\{\hat{\theta}_{n} \in[0,1]: \min _{i \in \mathcal{M}} \hat{t}_{n}^{i}\left(\hat{x} ; \vartheta_{n}^{i}, \hat{\theta}_{n}\right) \geq 0\right\} \neq \emptyset
$$

\footnotetext{
${ }^{25}$ Note that because of the nonsingularity of expression (36), the equivalent expression (37) is also nonsingular.
} 
for all $n \in \mathcal{N}$. The constants $\bar{\theta}_{n}$ in Theorem 3 are therefore well defined and by Berge's (1963, p. 116) maximum theorem condition (PM) is satisfied for all $\theta_{n} \in\left[0, \bar{\theta}_{n}\right]$. Hence, we have shown that given any efficient outcome $\hat{x}$, the pair $(\hat{t}, \hat{x})$ with $\hat{t}_{n}^{m}$ as defined in (21) constitutes a WTE, which concludes our proof.

Proof of Theorem 4. Let us first show that the excess transfer $\Delta=\left[\Delta_{n}^{m}\right]$ with $\Delta_{n}^{m}$ as defined in (25) solves (WT'). For this note that as a consequence of Assumption 2 we have that

$$
F^{m}(x) \leq \sum_{n \in \mathcal{N}} \frac{\partial F^{m}(\hat{x})}{\partial x_{n}} \cdot\left(x_{n}-\hat{x}_{n}\right)
$$

for any principal $m \in \mathcal{M}$ and any outcomes $x, \hat{x} \in \mathcal{X}$. Hence, using relations (27) and (38), it is

$$
\begin{aligned}
\sum_{n \in \mathcal{N}} \Delta_{n}^{m}(x) & =\sum_{n \in \mathcal{N}}\left(\frac{\partial F^{m}(\hat{x})}{\partial x_{n}} \cdot\left(x_{n}-\hat{x}_{n}\right)-\lambda_{n}^{m}(x) \sum_{j \neq n} G_{j}\left(x_{n}, \hat{x}_{-n}\right)\right) \\
& \geq 2 \sum_{n \in \mathcal{N}} \frac{\partial F^{m}(\hat{x})}{\partial x_{n}} \cdot\left(x_{n}-\hat{x}_{n}\right)-F^{m}(x) \geq F^{m}(x),
\end{aligned}
$$

for any outcome $x \in \mathcal{X}$ and any principal $m \in \mathcal{M}$ (relative to the efficient outcome $\hat{x} \in \mathcal{X}$ ). Hence, the inequality (WT') is satisfied on $\mathcal{X}$ for all $m \in \mathcal{M}$. We now turn our attention to (AM'). Using the normalization condition (26) and relation (38) we find that

$$
\begin{aligned}
\sum_{m \in \mathcal{M}} \Delta_{n}^{m}\left(x_{n}, \hat{x}_{-n}\right)-G\left(x_{n}, \hat{x}_{-n}\right) & =\sum_{m \in \mathcal{M}} \frac{\partial F^{m}(\hat{x})}{\partial x_{n}} \cdot\left(x_{n}-\hat{x}_{n}\right)-\sum_{j \in \mathcal{N}} G_{j}\left(x_{n}, \hat{x}_{-n}\right) \\
& \leq \sum_{m \in \mathcal{M}} F^{m}\left(x_{n}, \hat{x}_{-n}\right)-\sum_{j \in \mathcal{N}} G_{j}\left(x_{n}, \hat{x}_{-n}\right) \leq 0 .
\end{aligned}
$$

The last inequality follows from the definition of an efficient outcome $\hat{x}$. Hence, the inequality (AM') holds on $\mathcal{X}_{n}$ for all $n \in \mathcal{N}$.

Proof of Theorem 5. The solution (25) to the reduced contract-design problem (R) is well defined as long as there exist $M \cdot N$ functions $\lambda_{n}^{m} \in C(\mathcal{X}, \mathbb{R})$ that satisfy the $M+N$ relations (26) and (27). If (given the efficient outcome $\hat{x}$ ) for any $m \in \mathcal{M}$ we let

$$
\rho^{m}(x) \equiv F^{m}(x)-\left(\sum_{j \in \mathcal{N}} \frac{\partial F^{m}(\hat{x})}{\partial x_{j}} \cdot\left(x_{j}-\hat{x}_{j}\right)\right),
$$

then relations (26) and (27) can be rewritten equivalently in the form

$$
\left\{\begin{array}{rlr}
\beta^{m}(x) & \geq \rho^{m}(x), \quad m \in\{1, \ldots, M-1\} \\
-\sum_{n \in \mathcal{N}} \sum_{j \neq n} G_{j}\left(x_{n}, \hat{x}_{-n}\right) & \geq \rho^{M}(x)+\sum_{i=1}^{M-1} \beta^{i}(x) &
\end{array}\right.
$$

for all $x \in \mathcal{X}$, where

$$
\beta^{m}(x) \equiv-\sum_{n \in \mathcal{N}} \lambda_{n}^{m}(x)\left(\sum_{j \neq n} G_{j}\left(x_{n}, \hat{x}_{-n}\right)\right)
$$

for all $m \in\{1, \ldots, M-1\}$. Clearly, if $M-1$ functions $\beta^{1}, \ldots, \beta^{M-1} \in C(\mathcal{X}, \mathbb{R})$ can be found such that (40) holds for all $x \in \mathcal{X}$, then there also exist $\left(\lambda_{1}^{m}, \ldots, \lambda_{N}^{m}\right)$ for all $m \in\{1, \ldots, M-1\}$ such that (41) holds. ${ }^{26}$ In addition, if a solution to (40) exists, then $\beta^{m}=\rho^{m}$ for $m \in\{1, \ldots, M-1\}$ must also be a solution. A necessary and sufficient condition for the existence of a solution to (40) is therefore that

$$
\sum_{m \in \mathcal{M}} \rho^{m}(x)+\sum_{n \in \mathcal{N}} \sum_{j \neq n} G_{j}\left(x_{n}, \hat{x}_{-n}\right) \leq 0
$$

for all $x \in \mathcal{X}$. Using (39) relation (42) (on $\mathcal{X}$ ) is equivalent to (28), which concludes our proof.

\footnotetext{
${ }^{26}$ If for some $x \in \mathcal{X}$ the excess compliance cost $\sum_{j \neq n} G_{j}\left(x_{n}, \hat{x}_{-n}\right)$ vanishes for all $n \in \mathcal{N}$, then by virtue of inequality (38) in the proof of Theorem 4 the functions $\beta^{1}, \ldots, \beta^{M-1}$ with $\left(\beta^{1}, \ldots, \beta^{M-1}\right)=0$ solve $(40)$.
} 